\title{
MARK4 controls ischaemic heart failure through microtubule detyrosination
}

https://doi.org/10.1038/s41586-021-03573-5

Received: 15 December 2019

Accepted: 21 April 2021

Published online: 26 May 2021

Check for updates

\section{Xian $\mathrm{Yu}^{1,12}$, Xiao Chen ${ }^{2,12}$, Mamta Amrute-Nayak ${ }^{3}$, Edward Allgeyer ${ }^{4,5}$, Aite Zhao ${ }^{6}$, Hannah Chenoweth', Marc Clement', James Harrison', Christian Doreth', George Sirinakis ${ }^{4,5}$, Thomas Krieg ${ }^{7}$, Huiyu Zhou ${ }^{8}$, Hongda Huang ${ }^{9}$, Kiyotaka Tokuraku ${ }^{10}$, Daniel St Johnston ${ }^{4,5}$, Ziad Mallat ${ }^{1,11}$ \& Xuan Li ${ }^{1 凶}$}

\begin{abstract}
Myocardial infarction is a major cause of premature death in adults. Compromised cardiac function after myocardial infarction leads to chronic heart failure with systemic health complications and a high mortality rate ${ }^{1}$. Effective therapeutic strategies are needed to improve the recovery of cardiac function after myocardial infarction. More specifically, there is a major unmet need for a new class of drugs that can improve cardiomyocyte contractility, because inotropic therapies that are currently available have been associated with high morbidity and mortality in patients with systolic heart failure $^{2,3}$ or have shown a very modest reduction of risk of heart failure ${ }^{4}$. Microtubule detyrosination is emerging as an important mechanism for the regulation of cardiomyocyte contractility ${ }^{5}$. Here we show that deficiency of microtubule-affinity regulating kinase 4 (MARK4) substantially limits the reduction in the left ventricular ejection fraction after acute myocardial infarction in mice, without affecting infarct size or cardiac remodelling. Mechanistically, we provide evidence that MARK4 regulates cardiomyocyte contractility by promoting phosphorylation of microtubule-associated protein 4 (MAP4), which facilitates the access of vasohibin 2 (VASH2) - a tubulin carboxypeptidase-to microtubules for the detyrosination of $\alpha$-tubulin. Our results show how the detyrosination of microtubules in cardiomyocytes is finely tuned by MARK4 to regulate cardiac inotropy, and identify MARK4 as a promising therapeutic target for improving cardiac function after myocardial infarction.
\end{abstract}

Myocardial infarction-the main cause of ischaemic heart disease and chronic heart failure-is a serious ischaemic syndrome in which the blood supply to the heart is blocked, thus causing substantial death of myocardial cells and loss of function in the remaining viable cells ${ }^{6}$. Microtubule detyrosination, which is associated with desmin at force-generating sarcomeres ${ }^{5}$, is upregulated in failing hearts of patients with ischaemic cardiomyopathy ${ }^{5,7}$ and hypertrophic cardiomyopathies $^{5,7,8}$, and suppression of microtubule detyrosination improves contractility in failing cardiomyocytes ${ }^{7}$. VASH1 or VASH2, coupled to a small vasohibin-binding protein (SVBP), forms tubulin carboxypeptidases (TCPs) that are capable of tubulin detyrosination ${ }^{9,10}$. Depletion of VASH1 increases the speed of contraction and relaxation in failing human cardiomyocytes ${ }^{11}$. Structural and biophysical studies have suggested that VASH interacts with the $\mathrm{C}$-terminal tail of $\alpha$-tubulin ${ }^{12-14}$. However, the regulatory mechanisms of this system are still poorly understood.

Microtubule stability is regulated by microtubule-associated proteins (MAPs), including classical MAPs such as MAP2, MAP4 and tau $^{15}$. MAP4 is expressed in cardiomyocytes and the level of MAP4 significantly increases in human hearts with cardiomyopathy ${ }^{7}$. MAP4 dephosphorylation on the microtubule network has previously been described in a feline model of pressure-overload cardiac hypertrophy ${ }^{16}$, but the relationship between MAP4 phosphorylation and microtubule detyrosination has not been examined. MARK4 is an evolutionarily conserved serine-threonine kinase ${ }^{17,18}$ that is known to phosphorylate MAPs including tau, MAP2 and MAP4, on KXGS motifs within their microtubule-binding repeats ${ }^{19-21}$. The phosphorylation of MAPs triggered by MARK induces conformational changes that alter the association of MAPs with microtubules, and thereby regulates microtubule dynamics ${ }^{19-21}$. MARK4 is expressed in the heart ${ }^{20}$; however, the role of MARK4 in the cardiomyocyte has not been studied. Here we examined whether MARK4 regulates the function of the failing cardiomyocyte through modulation of microtubule detyrosination.

\section{Function of Mark4 $^{-/-}$hearts after myocardial infarction}

To evaluate the effect of MARK4 in the setting of ischaemic heart disease, we used a mouse model of permanent left anterior descending

1Department of Medicine, Cardiovascular Division, University of Cambridge, Addenbrooke's Hospital, Cambridge, UK. ${ }^{2}$ Department of Cardiology, Union Hospital, Tongji Medical College, Huazhong University of Science and Technology, Wuhan, China. ${ }^{3}$ Department of Molecular and Cell Physiology, Hannover Medical School, Hannover, Germany. ${ }^{4}$ The Gurdon Institute,

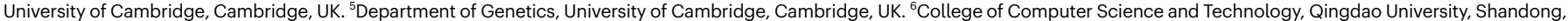
China. ${ }^{7}$ Department of Medicine, Experimental Medicine and Immunotherapeutics Division, University of Cambridge, Addenbrooke's Hospital, Cambridge, UK. ${ }^{8}$ School of Informatics, University of Leicester, Leicester, UK. ${ }^{9}$ Department of Biology, Southern University of Science and Technology, Shenzhen, China. ${ }^{10}$ Muroran Institute of Technology, Muroran, Japan.

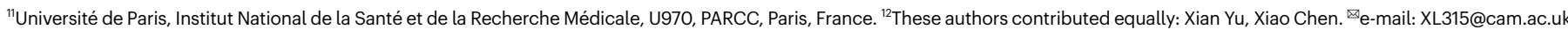



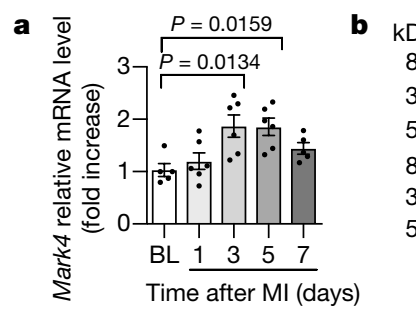

d
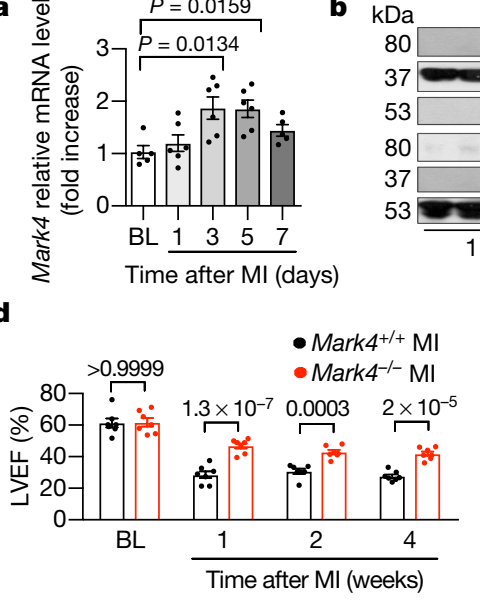

Fig. 1 | MARK4 deficiency preserves cardiac function after myocardial infarction without altering the size of the scar. a, Expression of Mark4 mRNA in heart samples from wild-type hearts, baseline hearts (BL; hearts without myocardial infarction) and from hearts obtained at the indicated days $(1,3$, 5 and 7 days) after myocardial infarction (MI) was analysed using real-time PCR. $n=5$ at baseline, $n=6$ mice per time point at 1,3 and 5 days after myocardial infarction and $n=5$ mice at 7 days after myocardial infarction. b. Western blots of wild-type hearts after myocardial infarction. Expression of MARK 4 in the insoluble cytoskeletal fractions (with desmin as marker) and GAPDH in corresponding soluble cytosolic fractions is shown. $n=3$ mice at each time

coronary artery ligation to induce a large myocardial infarction ${ }^{22,23}$ (Extended Data Fig. 1a). We detected Mark4 mRNA (Fig. 1a) and MARK4 protein (Fig. 1b) expression in the heart tissues, peaking between day 3 and day 5 after myocardial infarction (Fig. 1a-c). MARK4 was almost exclusively detected in the cytoskeleton-enriched insoluble fraction of the whole-heart extracts (Fig. 1b) and was localized in cardiomyocytes (Fig. 1c and Extended Data Fig. 2a). MARK4-deficient mice (Mark4 ${ }^{-{ }^{-}}$) displayed a remarkable preservation of left ventricular ejection fraction (LVEF), which was $63.6 \%$ ( $\pm 5.8 \%)$ higher compared with their wild-type littermate controls on the first week after left anterior descending coronary artery ligation (Fig. 1d), without any alteration in cardiac remodelling (Supplementary Table 1). Notably, infarct scar size was similar between the two groups of mice (Fig. 1e), indicating that the substantial difference in cardiac function between wild-type and $\mathrm{Mark}^{-/-}$mice was not attributable to differences in the size of viable cardiac tissues.

\section{MARK4 regulates cardiac contractility}

We found that the protective effect of MARK4 deficiency on the preservation of cardiac function was already apparent at $24 \mathrm{~h}$ after myocardial infarction (Fig. 2a and Extended Data Fig. 1b), despite a similar extent of myocardial injury, shown by comparable serum levels of cardiac troponin I (Fig. 2b) and a comparable infarct size analysed by triphenyltetrazolium chloride staining (Fig. 2c), in $\mathrm{Mark}^{-{ }^{--}}$and wild-type mice. MARK4 has previously been shown to regulate NLRP3 activation in macrophage ${ }^{24,25}$, which could affect the outcome of a post-ischaemic injury given the role of the NLRP3 inflammasome in this setting ${ }^{26,27}$. However, MARK4 deficiency did not significantly alter local and systemic inflammatory responses to myocardial injury at day 3 after myocardial infarction (Extended Data Fig. 2b and Supplementary Table 2) when the preservation of the LVEF was already evident in Mark4 $^{-1-}$ mice (Extended Data Fig. 2c). Moreover, bone marrow transfer of $\mathrm{Mark}^{-/-}$haematopoietic cells into wild-type mice (Extended Data Fig. 1c; validation in Extended Data Fig. 3a, b) did not improve cardiac function after myocardial infarction in comparison with the transfer of wild-type bone marrow cells (Fig. 2d), indicating that the protective c

C Baseline
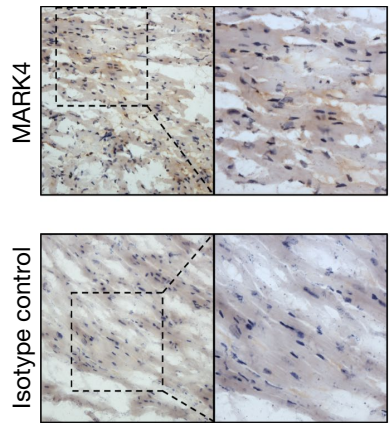

Day 3 after MI
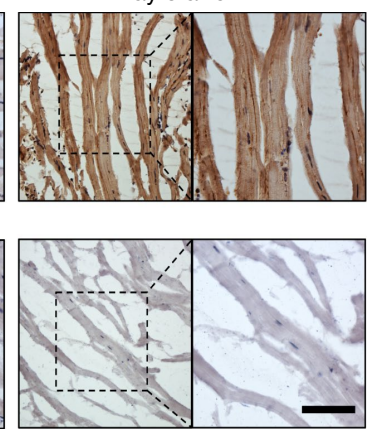

point. c, Representative immunohistochemistry staining of MARK4 in wild-type mice at baseline or after myocardial infarction. Isotype IgG was used as control. Scale bar, $50 \mu \mathrm{m}$. d, Assessment of LVEF in Mark4 $4^{-1-}$ mice $(n=7)$ and their littermate controls $\left(\right.$ Mark $\left.^{+/+}\right)(n=7)$ at baseline, and 1, 2 and 4 weeks after myocardial infarction. e, Scar size at week 4 after myocardial infarction. Scale bar, $2 \mathrm{~mm}$. a, d, e, Data are mean \pm s.e.m. One-way analysis of variance (ANOVA) with Bonferroni post hoc correction (a); two-way ANOVA with Bonferroni post hoc correction for multiple comparisons (d); two-tailed unpaired Student's $t$-test (e). $P$ values are indicated. effect of MARK4 deficiency after myocardial infarction could not be explained by the role of MARK4 in haematopoietic cells. By contrast, using an inducible conditional deletion of Mark4 in cardiomyocytes (Mark4 conditional knockout (cKO)) (Extended Data Fig. 1d; validation in Extended Data Fig. 3c), we found a substantial preservation of LVEF in Mark4 cKO mice after myocardial infarction, which was $56.8 \%$ $( \pm 6.2 \%)$ higher compared with littermate control mice at day 1 after myocardial infarction (Fig. 2e). The protective effect seen in Mark4 cKO mice started as early as the first day after myocardial infarction and lasted until the end of the observation at four weeks after myocardial infarction (Fig. 2e). Notably, Mark4 cKO mice had a reduction of only $4.3 \%( \pm 3.8 \%)$ in LVEF at day 1 after myocardial infarction, compared with a reduction of $37.9 \%( \pm 5.5 \%)$ in the control mice (Fig. $2 \mathrm{e}$ ), without any difference in infarct size (Extended Data Fig. 3e). The data further show that the remaining, viable MARK4-deficient cardiomyocytes affect contractile function. Collectively, our data demonstrate that cardiomyocyte-expressed MARK4 has an intrinsic role in the control of cardiac function after myocardial infarction.

To examine the effect of MARK4 on cardiomyocyte function, we subjected freshly isolated primary cardiomyocyte ${ }^{28}$ from wild-type and $\mathrm{Mark}^{-/-}$mice to a single-cell contractility assay using an electrical stimulator (Fig. $2 \mathrm{f}-\mathrm{i}$ ). We found that sarcomere peak shortening of isolated cardiomyocytes strongly correlated with the in vivo LVEF (Fig. 2f), indicating that the contraction of isolated cardiomyocyte measured ex vivo reflects LVEF assessed in vivo (Figs.1d, 2a, e). At baseline, wild-type and MARK4-deficient cardiomyocytes had similar levels of resting sarcomere length (Extended Data Fig. 4a,b), sarcomere peak shortening and contraction and relaxation velocities (Fig. $2 g-i$ ), an observation that is consistent with the absence of a difference in LVEF between wild-type and $\mathrm{Mark}^{-/-}$mice before myocardial infarction (Fig.1d). After myocardial infarction, wild-type cardiomyocytes displayed markedly reduced sarcomere shortening (decreased by $22.5 \% \pm 3.7 \%$ ) (Fig. $2 \mathrm{~g}$ and Extended Data Fig. 4c), with slower relaxation velocity (decreased by $25.2 \% \pm 4.4 \%$ ) (Fig. $2 \mathrm{i}$ and Extended Data Fig. $4 \mathrm{e}$ ), compared with cardiomyocytes isolated from wild-type mice without myocardial infarction. Notably, although no difference in resting sarcomere length 

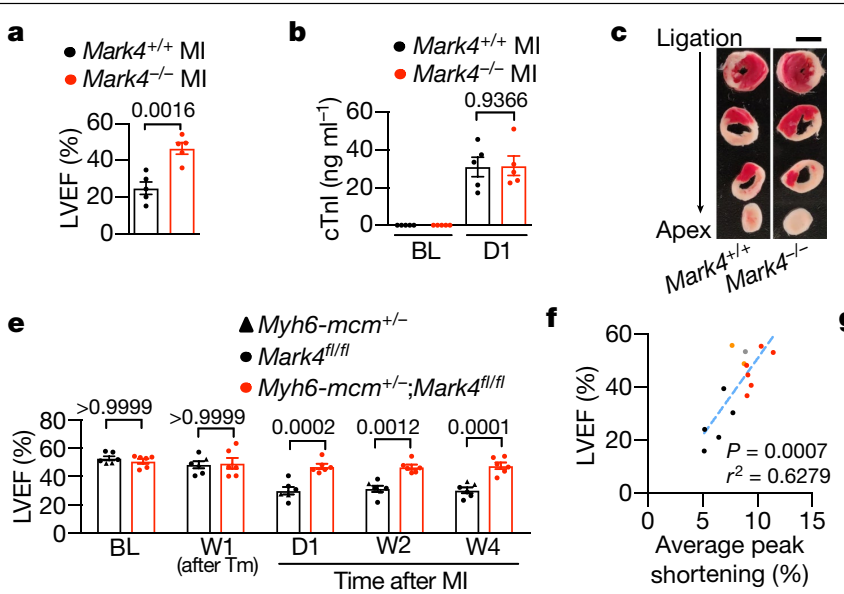

Fig. 2 | MARK4 expression in cardiomyocytes regulates cardiac contractile function after myocardial infarction. a-c, $\operatorname{Mark}^{-/-}$mice $(n=5)$ and their littermate controls (Mark4 $\left.{ }^{+/+}, n=5\right)$ at day 1 (D1) after myocardial infarction. a, LVEF. b, c, Circulating cardiac troponin I (cTnI) levels (b) and infarct size (c) at $24 \mathrm{~h}$ after myocardial infarction are shown. cardiac troponin I measurements at baseline were used as controls. Scale bar, $2 \mathrm{~mm}$. d, Assessment of LVEF in chimeric mice ( $n=8$ wild-type recipients of $\operatorname{Mark}^{+/+}$bone marrow (BM) donors; $n=6$ wild-type recipients of $\mathrm{Mark4}^{-/-}$bone marrow donors) at the indicated time points. e, Assessment of LVEF at the indicated time points after conditional Mark4 knockout using tamoxifen (Tm) in Myh6- $\mathrm{mcm}^{+/-} ;$Mark $^{\text {fl/fl }}$ (also known as $\alpha M H C-m_{c m}^{+/-}$;Mark ${ }^{f l f l}$ ) mice $(n=6)$ (Myh6 encodes the cardiomyocyte-specific marker $\alpha \mathrm{MHC})$. Tamoxifen-injected $\mathrm{Myh}^{-} \mathrm{mcm}^{+-}$and Mark $4^{f l f l}$ littermate mice were used as controls $(n=6)$. $\mathbf{f}-\mathbf{i}$, Contractility assay of single primary cardiomyocytes isolated at baseline or at day 3 after myocardial

was observed between Mark4 $^{-/-}$and wild-type cardiomyocytes after myocardial infarction (Extended Data Fig. 4b), Mark4 $^{-/-}$cardiomyocytes displayed a greater level of sarcomere shortening (increased by $36.0 \% \pm 6.0 \%$ ) (Fig. $2 \mathrm{~g}$ and Extended Data Fig. $4 \mathrm{~d}$ ) together with a greater velocity during both the contraction (increased by $42.0 \% \pm 6.9 \%$ ) and relaxation (increased by $46.7 \% \pm 7.5 \%$ ) phases (Fig. $2 \mathrm{~h}$, i and Extended Data Fig. 4f) compared with wild-type cardiomyocytes. Upstream changes in the influx of calcium $\left(\mathrm{Ca}^{2+}\right)$ through excitation-contraction coupling could contribute to the contractile alterations; however, we did not observe any significant difference in $\mathrm{Ca}^{2+}$ transients between electrically stimulated Mark4 $^{-/-}$and wild-type cardiomyocytes at baseline or at day 3 after myocardial infarction (Extended Data Fig. 4g-m). These data demonstrate that MARK4 deficiency substantially improves both contractile and relaxation functions of cardiomyocytes after myocardial infarction.

\section{MARK4 alters microtubule detyrosination}

Detyrosinated microtubules represent tunable, compression-resistant elements that impair cardiac function in failing hearts in humans ${ }^{5,7}$. We confirmed that the level of detyrosinated $\alpha$-tubulin was significantly higher in cardiomyocytes isolated from ischaemic hearts compared with cardiomyocytes isolated from mice that received a sham operation, in contrast to the remaining cell pool (immune cells, fibroblasts and endothelial cells), which did not display a change in $\alpha$-tubulin detyrosination (Extended Data Fig. 2d, e). Previous data indicated that MARK4 affects the posttranslational detyrosination and polyglutamylation of microtubules in ciliated cells ${ }^{29}$. Therefore, we hypothesized that MARK4 deficiency may affect microtubule detyrosination in cardiomyocytes after myocardial infarction. We found a significantly lower level of detyrosinated microtubules in whole-heart tissue extracts (Fig. 3a, b), and in isolated cardiomyocytes (together with reduced polyglutamylated microtubules) (Fig. 3e-g and Extended Data Fig. 2f, g) of Mark $^{-1-}$ mice compared with littermate wild-type controls after

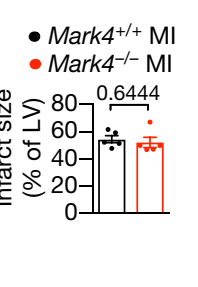

\section{d}
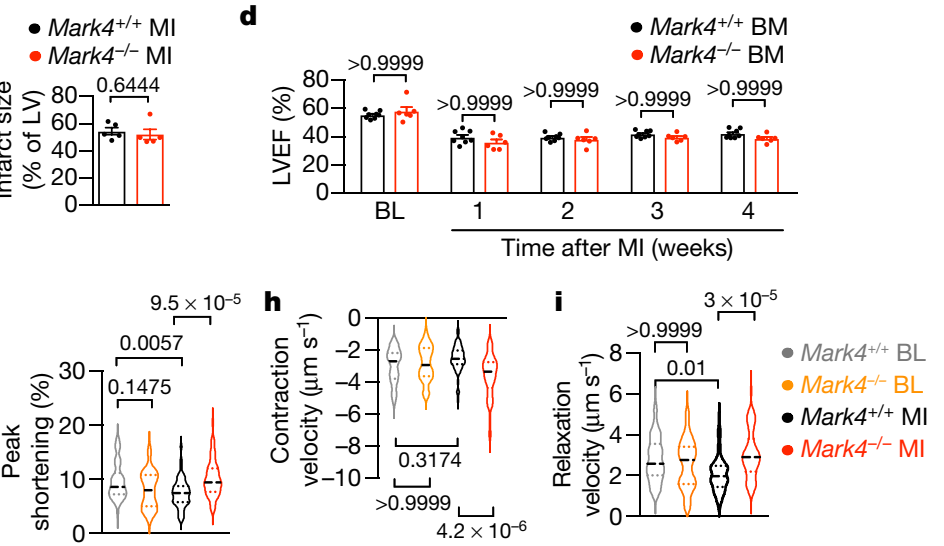

infarction in the following groups: baseline, $\operatorname{Mark}^{+/+}$mice $(n=4$ mice, $n=45$ cardiomyocytes examined over 4 independent experiments); baseline, Mark $4^{-/-}$mice ( $n=3$ mice, $n=45$ cardiomyocytes examined over 3 independent experiments); myocardial infarction, $\operatorname{Mark}^{+/+}$mice $(n=5$ mice, $n=54$ cardiomyocytes examined over 5 independent experiments); and myocardial infarction, Mark $^{-/-}$mice ( $n=6$ mice, $n=57$ cardiomyocytes examined over 6 independent experiments).f, Correlation between LVEF (measured at day 1 after myocardial infarction) and sarcomere peak shortening. g, Sarcomere peak shortening. $\mathbf{h}, \mathbf{i}$, Pooled data of contraction (h) and relaxation (i) velocity. $\mathbf{a}-\mathbf{e}$, Data are mean \pm s.e.m. $g-\mathbf{i}$, Violin plots show lines at the median (solid) and quartiles (dashed). Two-tailed unpaired $t$-test $(\mathbf{a}-\mathbf{c})$; two-way ANOVA with Bonferroni post hoc correction for multiple comparisons $(\mathbf{d}, \mathbf{e}, \mathbf{g}-\mathbf{i})$. $P$ values are indicated.

myocardial infarction. In the absence of MARK4, we observed a reduced ratio of $\alpha$-tubulin in the soluble fraction versus its level in the insoluble fraction (Fig. 3c), indicating a reduced percentage of free tubulin without MARK4. Notably, we found that the level of tubulin detyrosination inversely correlated with LVEF (Fig. 3d), suggesting that the MARK4-dependent modulation of microtubule detyrosination has an important role in controlling cardiac function after myocardial infarction.

To further address the hypothesis that MARK4 deficiency improves cardiomyocyte contractility through its influence on microtubule detyrosination, we used a genetic approach to overexpress tubulin tyrosine ligase (TTL) using an adenovirus system (Extended Data Fig. 5a-c) to reverse the effect of $\mathrm{TCP}^{30}$ (Fig. $\left.3 \mathrm{~h}-\mathrm{j}\right)$. TTL overexpression robustly improved peak shortening (Fig. 3h and Extended Data Fig. 5d) and increased the velocity of both contraction and relaxation (Fig. 3i,j and Extended Data Fig. 5g) of failing wild-type cardiomycytes ${ }^{7}$. However, overexpression of TTL could not further improve peak shortening (Fig. 3h and Extended Data Fig. 5e) and contractile velocities of $\mathrm{Mark4}^{-{ }^{-}}$ cardiomyocytes after myocardial infarction (Fig. 3i,j and Extended Data Fig. $5 \mathrm{~h}$ ), which is consistent with the already low level of detyrosinated microtubules in $\mathrm{Mark}^{-/-}$cardiomyocytes. We further confirmed these data using a pharmacological approach with parthenolide to inhibit microtubule detyrosination $^{5,7}$ (Extended Data Fig. $5 j-s$ ). Taken together, our data show that MARK4 regulates cardiac inotropic function through its effect on microtubule detyrosination in cardiomyocytes.

\section{MARK4 directs VASH2 access to microtubules}

Detyrosination of $\alpha$-tubulin preferentially occurs on polymerized microtubules ${ }^{31}$. Apart from binding to VASH, the C-terminal tubulin tails of polymerized microtubules are also important for MAP binding ${ }^{32,33}$. MAP4 bound to the $\mathrm{C}$-terminal tubulin tail along the protofilament stabilizes the longitudinal contacts of the microtubule, and this interaction can affect other microtubule-binding partners such as the motor 


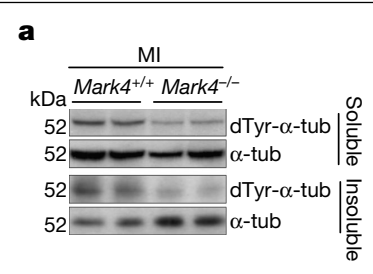

b $\mathrm{Mark}^{+/+} \mathrm{Ml}$ - Mark4 $4^{-/-} \mathrm{Ml}$ $\stackrel{0.0039}{0} 0.0036$
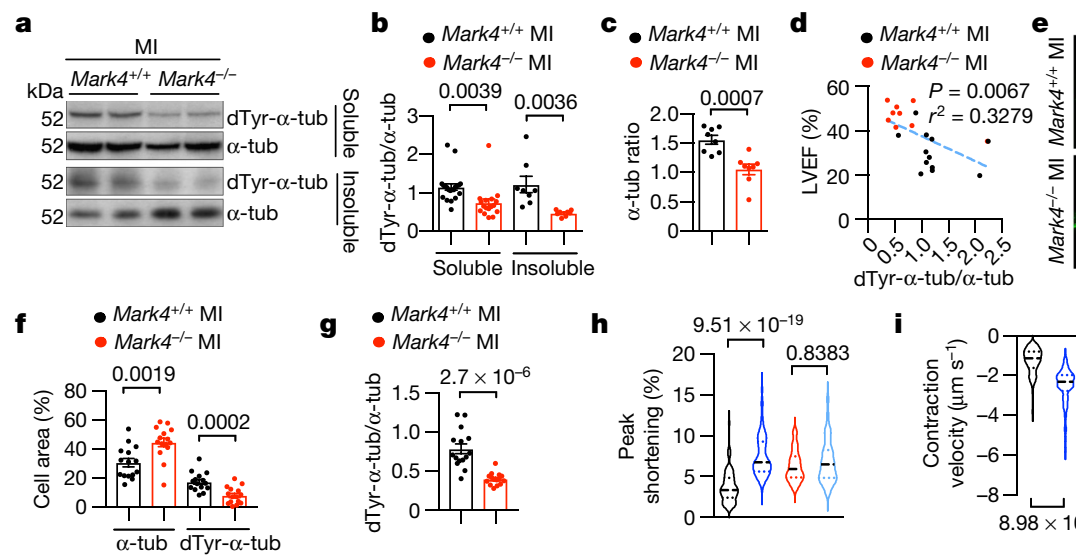

i
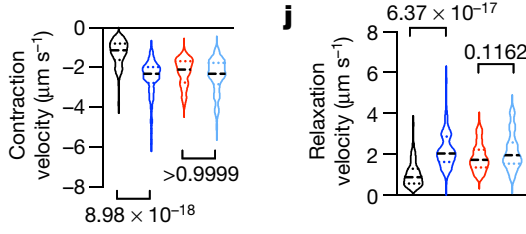

- Mark4 ${ }^{+/+} \mathrm{MI}$, Adv-null OE - Mark4 ${ }^{+/+}$MI, Adv-TTL OE - Mark4 ${ }^{-/-}$MI, Adv-null OE - Mark4 $4^{-/-}$MI, Adv-TTL OE
Fig. 3 | MARK4 regulates cardiomyocyte contractility by promoting microtubule detyrosination. a-d, Western blots of whole-heart extraction from mice at day 3 after myocardial infarction, in soluble and insoluble fractions. dTyr-tub, detyrosinated $\alpha$-tubulin; $\alpha$-tub, $\alpha$-tubulin.

a, Representative western blots. $\mathbf{b}$, Ratio of detyrosinated $\alpha$-tubulin over total $\alpha$-tubulin in the following groups: myocardial infarction, soluble fraction, $\operatorname{Mark4}^{+/+}$mice $(n=20)$; myocardial infarction, soluble fraction, Mark $^{-/-}$mice $(n=17)$; myocardial infarction, insoluble fraction, $\operatorname{Mark}^{+/+}$mice $(n=8)$; and myocardial infarction, insoluble fraction $\operatorname{Mark}^{-1-}$ mice $(n=8)$. c, Ratio of $\alpha$-tubulin in the soluble fraction over $\alpha$-tubulin in the insoluble fraction ( $n=8$ mice per group).d, Correlation between LVEF and the ratio of detyrosinated $\alpha$-tubulin/ $\alpha$-tubulin in $\operatorname{Mark4}^{-/-}(n=9)$ and control mice $(n=12)$. e-g, Confocal images of isolated cardiomyocytes at day 3 after myocardial infarction.e, Representative images. Scale bar, $20 \mu \mathrm{m} . \mathbf{f}, \mathbf{g}$, Percentage of detyrosinated $\alpha$-tubulin or total $\alpha$-tubulin area per cell $(\mathbf{f})$ and ratio of detyrosinated $\alpha$-tubulin/total $\alpha$-tubulin $(n=3$ mice, $n=15$ cardiomyocytes per group) (g). h-j, Adenovirus (Adv)-mediated overexpression (OE) of TTL in primary cardiomyocytes isolated from $\mathrm{Mark}^{-/-}$or control mice at day 3 after myocardial infarction, with overexpression of a null virus as controls. Contractility assay of single cardiomyocytes in the following groups: myocardial infarction, Adv-Null, Mark $^{+/+}$mice $(n=3$ mice, $n=75$ cardiomyocytes examined over 3 independent experiments); myocardial infarction, Adv-TTL, Mark4 ${ }^{+/+}$mice $(n=3$ mice, $n=69$ cardiomyocytes examined over 3 independent experiments); myocardial infarction, Adv-Null, Mark4 ${ }^{-/-}$ mice ( $n=3$ mice, $n=74$ cardiomyocytes examined over 3 independent experiments); and myocardial infarction, Adv-TTL, Mark $4^{-1-}$ mice $(n=3$ mice, $n=73$ cardiomyocytes examined over 3 independent experiments).

$\mathbf{h}$, Sarcomere peak shortening. Pooled data of contraction (i) and relaxation ( $\mathbf{j})$ velocity.h-j, Violin plots show lines at the median (solid) and quartiles (dashed).b, c, f, g, Data are mean \pm s.e.m. Two-tailed unpaired $t$-test $(\mathbf{b}, \mathbf{c}, \mathbf{f}, \mathbf{g})$; two-tailed correlation test (d); two-way ANOVA with Bonferroni post hoc correction for multiple comparisons $(\mathbf{h}-\mathbf{j})$. Pvalues are indicated. protein kinesin- $1^{33}$. MARK4, as a kinase, is expected to phosphorylate MAP4 at its KXGS motifs (including S941 and S1073 in human MAP4, or S914 and S1046 in mouse MAP4) within its microtubule-binding repeats ${ }^{19,20}$ (Extended Data Fig. 6a) and alter MAP4-binding status on the protofilament (Extended Data Fig. 6b). We therefore hypothesized that MARK4-by modifying MAP4 phosphorylation-may affect VASH accessibility to the $\mathrm{C}$-terminal $\alpha$-tubulin tail and therefore influence microtubule detyrosination. As such, we used an in vitro microtubule co-sedimentation assay. Both MAP4 (Extended Data Fig. 6c, d) and VASH2-SVBP (Extended Data Fig. 6e, f) were able to incrementally bind to polymerized microtubules when incremental amounts were separately applied in the assays, which is consistent with the results of previous studies ${ }^{12,33}$. Notably, we found that VASH2-SVBP bound to polymerized microtubules gradually decreased in the presence of incremental amounts of previously bound MAP4 (with four microtubule-binding repeats (4R-MAP4)) (Fig. 4a, b). Therefore, these results support the hypothesis that the level of MAP4 occupancy on the polymerized microtubules influences the level of access of VASH2 to the microtubule protofilaments.

To confirm this hypothesis in vivo, we performed biochemical subcellular fractionation on primary cardiomyocytes isolated from non-ischaemic and ischaemic hearts of wild-type and $\mathrm{Mark}^{-/-}$mice using a commercial kit, which we have validated (Extended Data Fig. 7a, b). We first confirmed that MAP4 was expressed in the cardiomyocytes and that the level of MAP4 was higher after myocardial infarction (Extended Data Fig. 7c), a result consistent with data showing that MAP4 levels significantly increase in hearts of individuals with cardiomyopathies ${ }^{7}$. MAP4 was detected in its S914 phosphorylated form ((pMAP4(S914); S914 is located within the KXGS motif)) in the pellet extraction buffer (PEB) and also in its S1046 form (pMAP4(S1046); S1046 is located within the KXGS motif) in the cytosolic extraction buffer (CEB) (Extended Data Fig. 7c-e). Knocking down MAP4 using small hairpin RNA (shRNA) in isolated cardiomyocytes after myocardial infarction led to increased
VASH2 levels in the PEB fraction, which was confirmed by both western blot and immunocytochemistry (Extended Data Fig. 7f-i); these results are in line with the results of an in vitro microtubule co-sedimentation assay (Fig. 4a, b). VASH2 was detected as a specific band (validated by specific knockdown using shRNA) (Extended Data Fig. 8a) of around $50 \mathrm{kDa}$ in the PEB fraction (Extended Data Fig. 8a, b), which is higher than its theoretical molecular weight of $40 \mathrm{kDa}$ and presumably due to the formation of a stable complex with SVBP, because the addition of a denaturing agent (urea) reduced its size to around $40 \mathrm{kDa}$ (Extended Data Fig. 8b). After myocardial infarction, pMAP4(S914) was detected in a $110 \mathrm{kDa}$ form in the PEB fraction whereas pMAP4(S1046) was detected in a $220 \mathrm{kDa}$ form in the CEB fraction (Fig. 4c and Extended Data Fig. 7c). MAP4 was detected as giant puncta in the cytosol of cardiomyocytes isolated after myocardial infarction, and these puncta were barely present at baseline (Extended Data Fig. 8c, d). pMAP4(S1046) (in the CEB fraction) formed oligomerized structures (at $440 \mathrm{kDa}$ or higher) as revealed by native gel analysis (Extended Data Fig. 8e, f), and these pMAP4(S1046) oligomers could be further reduced to the $220 \mathrm{kDa}$ form in the presence of urea as revealed by denaturing gel analysis (Extended Data Fig. 8g). The data suggest that MAP4 phosphorylation at S1046 is associated with its presence as oligomers or giant puncta in the cytosol in situ. Our results are consistent with a structural model, in which $\mathrm{S} 914$ is within the weak binding site of the microtubule-binding repeat of MAP4 to the microtubules, whereas S1046 is within the strong anchor point of the microtubule-binding repeat of MAP4 to the microtubules ${ }^{33}$ (Extended Data Fig. 6b), so that phosphorylation at S1046 leads to the detachment of MAP4 from polymerized microtubules and accumulation in the cytosol. Accordingly, a higher level of pMAP4(S1046) was strongly and positively correlated with increased VASH2 levels in the PEB fraction (there was also a weaker correlation between pMAP4(S914) levels and VASH2 levels in the PEB fraction) (Extended Data Fig. 7c, e) in wild-type cardiomyocytes, indicating an association between pMAP4 (phosphorylated at S941 and S1046) and VASH2 levels on 


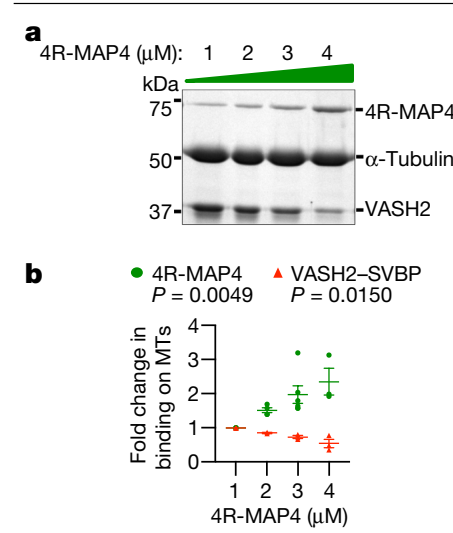

c

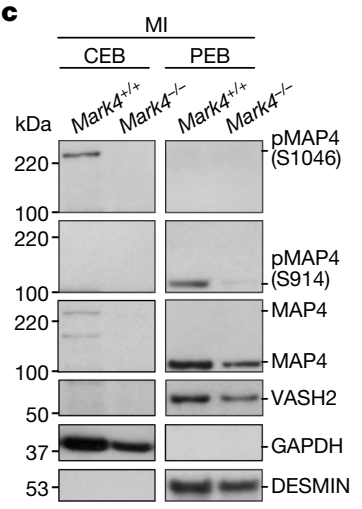

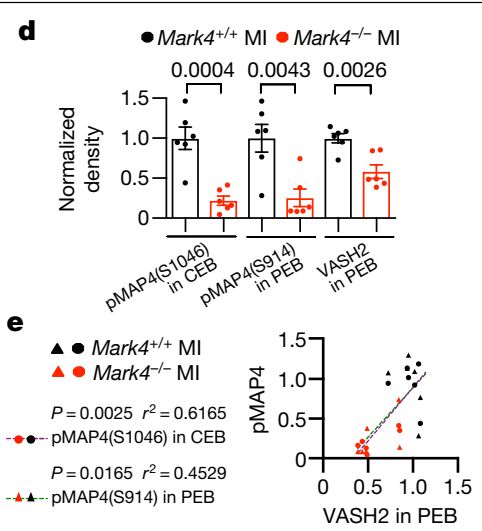

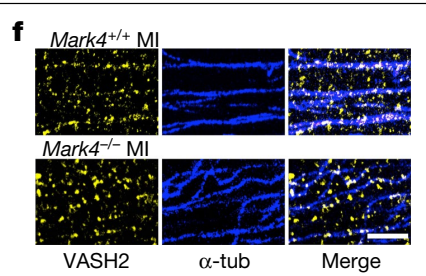

g $\mathrm{Mark}^{+/+} \mathrm{Ml}$

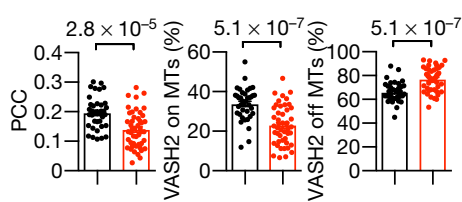

Fig. 4 | MARK4 controls microtubule detyrosination through MAP4 phosphorylation to facilitate VASH2 access to microtubules. a, b, Representative gel image of VASH2-SVBP $(3 \mu \mathrm{M})$ binding to polymerized microtubules (MTs) $(5 \mu \mathrm{M})$ in the presence of different amounts of 4R-MAP4 $(1-4 \mu \mathrm{M})$ in a microtubule co-sedimentation assay (a) and quantification of the binding (b). $n=3$ independent experiments per group. $\mathbf{c}-\mathbf{e}$, Subcellular fractionations of $\mathrm{Mark4}^{-/-}$or control cardiomyocytes isolated after myocardial infarction. c, Representative western blots of the fractions from CEB or PEB derived from the same experiment. d, Quantification of the levels of pMAP4(S1046) in CEB, pMAP4(S914) in PEB and VASH2 in PEB ( $n=6$ mice per group, blots were processed in parallel). e, Correlation between VASH2 level in the PEB fraction and pMAP4 levels. $\mathbf{f}, \mathbf{g}$, Stimulated emission depletion images of VASH 2 and $\alpha$-tubulin in $\mathrm{Mark}^{-/-}$or control cardiomyocytes isolated from mice after myocardial infarction.f, Representative images. Scale bar, $2 \mu \mathrm{m}$. g, Pearson correlation coefficient (PCC) of VASH 2 and $\alpha$-tubulin signals, percentage of VASH2 signals on the polymerized microtubules, percentage of VASH 2 signals off the polymerized microtubules in the $\mathrm{Mark}^{+/+}$myocardial infarction group ( $n=6$ mice, $n=38$ cardiomyocytes examined over 3 independent experiments) and $\mathrm{Mark}^{-/-}$myocardial infarction group ( $n=6$ mice, $n=47$ cardiomyocytes examined over 3 independent experiments). b, $\mathbf{d}, \mathbf{g}$, Data are mean \pm s.e.m. One-way ANOVA (b); two-tailed unpaired $t$-test $(\mathbf{d}, \mathbf{g})$; two-tailed correlation test (e). $P$ values are indicated. the polymerized microtubules. Notably, levels of pMAP4(S914) and pMAP4(S1046) were substantially reduced in Mark $^{-/-}$cardiomyocytes after myocardial infarction (Fig. 4c, d), confirming that S914 and S1046 of MAP4 are substrate sites of the MARK4 kinase. Reduced levels of pMAP4(S1046) in the CEB fraction and pMAP4(S914) in the PEB fraction correlated well with a reduced level of VASH2 in the PEB fraction $\left(r^{2}=0.6165, P=0.0025 ; r^{2}=0.4529, P=0.0165\right.$, respectively) $($ Fig. $4 \mathrm{c}-\mathrm{e})$, with a stronger association between pMAP4(S1046) and VASH2. In addition, we found that VASH2 levels were positively correlated with desmin levels in the PEB fraction (Extended Data Fig. $8 \mathrm{~h}-\mathrm{j}$ ), supporting previous data that detyrosinated microtubules are positively correlated with desmin levels in cardiomyocytes ${ }^{5}$. In summary, our results suggest that MARK4 kinase, through phosphorylation of MAP4 at S914 and S1046, changes the status of MAP4 to allow VASH2 access to the polymerized microtubule for its TCP activity.

To further confirm the causal effect of MARK4 on VASH2 localization, we overexpressed MARK4 in primary cardiomyocytes, which caused the appearance of pMAP4(S1046) (Extended Data Fig. 9a-c) and giant MAP4 puncta in the cytosol (Extended Data Fig. 9d,e) and led to increased VASH2 levels in the PEB fraction (Extended Data Fig. 9a-c). By using stimulated emission depletion super-resolution microscopy ${ }^{34}$, we found a strong localization of VASH 2 on the polymerized microtubules in primary cardiomyocytes isolated from wild-type hearts after myocardial infarction compared with samples isolated from wild-type hearts at baseline (Extended Data Fig. 10a, b). Total VASH2 levels were comparable between Mark4 $^{-/-}$and $\mathrm{Mark4}^{+/+}$cardiomyocytes after myocardial infarction (Extended Data Fig. 10c, d). However, there was a significant reduction in the association between VASH2 and polymerized microtubules in $\mathrm{Mark}^{-/-}$compared with wild-type cardiomyocytes (Fig. 4f, g). In conclusion, our results demonstrate that MARK4 regulates microtubule detyrosination by phosphorylating MAP4 and controlling VASH2 accessibility to the microtubules (Extended Data Fig. 10e).

\section{Discussion}

Detyrosinated microtubules interfere with the contractile function of cardiomyocytes from failing hearts in humans ${ }^{7}$, and targeting the regulatory mechanism that controls microtubule detyrosination could represent a new inotropic strategy for improving cardiac function. We show that MARK4 has an important role in the alteration of cardiomyocyte contractility through the modulation of microtubule detyrosination in the ischaemic heart. It will be interesting to examine whether this protective effect of MARK4 inactivation on cardiac function after myocardial infarction is sustained in the very long term (several months after myocardial infarction) without inducing any harmful side effects, and whether MARK4 inhibition can improve contractile function in the setting of non-ischaemic heart failure. Furthermore, the marked improvement in relaxation kinetics in the absence of MARK4 raises the possibility of a potential beneficial effect of MARK4 inhibition in the setting of heart failure with preserved ejection fraction, an increasingly common cardiac syndrome that is associated with high morbidity and mortality. The molecular and structural mechanisms of MARK 4 coupled with MAP4 and VASH2-SVBP in modifying microtubule detyrosination will need to be investigated in other settings such as mitosis, where regulation of detyrosinated microtubules has important pathophysiological relevance ${ }^{9,35}$, and the differential role of other TCPs (for example, VASH1) will need to be studied further in future.

\section{Online content}

Any methods, additional references, Nature Research reporting summaries, source data, extended data, supplementary information, acknowledgements, peer review information; details of author contributions and competing interests; and statements of data and code availability are available at https://doi.org/10.1038/s41586-021-03573-5.

1. Murray, C. J. \& Lopez, A. D. Measuring the global burden of disease. N. Engl. J. Med. 369 448-457 (2013).

2. Packer, M. et al. Effect of oral milrinone on mortality in severe chronic heart failure. N. Engl. J. Med. 325, 1468-1475 (1991).

3. Cohn, J. N. et al. A dose-dependent increase in mortality with vesnarinone among patients with severe heart failure. N. Engl. J. Med. 339, 1810-1816 (1998).

4. Teerlink, J. R. et al. Cardiac myosin activation with omecamtiv mecarbil in systolic heart failure. N. Engl. J. Med. 384, 105-116 (2021).

5. Robison, P. et al. Detyrosinated microtubules buckle and bear load in contracting cardiomyocytes. Science 352, aaf0659 (2016). 
6. Anderson, J. L. \& Morrow, D. A. Acute myocardial infarction. N. Engl. J. Med. 376, 2053-2064 (2017).

7. Chen, C. Y. et al. Suppression of detyrosinated microtubules improves cardiomyocyte function in human heart failure. Nat. Med. 24, 1225-1233 (2018).

8. Schuldt, M. et al. Proteomic and functional studies reveal detyrosinated tubulin as treatment target in sarcomere mutation-induced hypertrophic cardiomyopathy. Circ. Heart Fail. 14, e007022 (2021).

9. Aillaud, C. et al. Vasohibins/SVBP are tubulin carboxypeptidases (TCPs) that regulate neuron differentiation. Science 358, 1448-1453 (2017).

10. Nieuwenhuis, J. et al. Vasohibins encode tubulin detyrosinating activity. Science $\mathbf{3 5 8}$, 1453-1456 (2017).

11. Chen, C. Y. et al. Depletion of vasohibin 1 speeds contraction and relaxation in failing human cardiomyocytes. Circ. Res. 127, e14-e27 (2020).

12. Wang, N. et al. Structural basis of tubulin detyrosination by the vasohibin-SVBP enzyme complex. Nat. Struct. Mol. Biol. 26, 571-582 (2019).

13. Li, F., Hu, Y., Qi, S., Luo, X. \& Yu, H. Structural basis of tubulin detyrosination by vasohibins. Nat. Struct. Mol. Biol. 26, 583-591 (2019).

14. Zhou, C., Yan, L., Zhang, W. H. \& Liu, Z. Structural basis of tubulin detyrosination by VASH2/SVBP heterodimer. Nat. Commun. 10, 3212 (2019).

15. Ramkumar, A., Jong, B. Y. \& Ori-McKenney, K. M. Remapping the microtubule landscape: how phosphorylation dictates the activities of microtubule-associated proteins. Dev. Dyn. 247, 138-155 (2018).

16. Chinnakkannu, P. et al. Site-specific microtubule-associated protein 4 dephosphorylation causes microtubule network densification in pressure overload cardiac hypertrophy. J. Biol. Chem. 285, 21837-21848 (2010).

17. Doerflinger, H., Benton, R., Shulman, J. M. \& St Johnston, D. The role of PAR-1 in regulating the polarised microtubule cytoskeleton in the Drosophila follicular epithelium. Development 130, 3965-3975 (2003).

18. Goldstein, B. \& Macara, I. G. The PAR proteins: fundamental players in animal cell polarization. Dev. Cell 13, 609-622 (2007).

19. Illenberger, S. et al. Phosphorylation of microtubule-associated proteins MAP2 and MAP4 by the protein kinase $1110^{\text {mark }}$. Phosphorylation sites and regulation of microtubule dynamics. J. Biol. Chem. 271, 10834-10843 (1996).

20. Trinczek, B., Brajenovic, M., Ebneth, A. \& Drewes, G. MARK4 is a novel microtubule-associated proteins/microtubule affinity-regulating kinase that binds to the cellular microtubule network and to centrosomes. J. Biol. Chem. 279, 5915-5923 (2004).

21. Drewes, G., Ebneth, A. \& Mandelkow, E. M. MAPs, MARKs and microtubule dynamics. Trends Biochem. Sci. 23, 307-311 (1998).
22. Zouggari, Y. et al. B lymphocytes trigger monocyte mobilization and impair heart function after acute myocardial infarction. Nat. Med. 19, 1273-1280 (2013).

23. Pell, V. R. et al. Ischemic preconditioning protects against cardiac ischemia reperfusion injury without affecting succinate accumulation or oxidation. J. Mol. Cell. Cardiol. 123, 88-91 (2018).

24. Li, X. et al. MARK4 regulates NLRP3 positioning and inflammasome activation through a microtubule-dependent mechanism. Nat. Commun. 8, 15986 (2017).

25. Clement, M. et al. MARK4 (microtubule affinity-regulating kinase 4)-dependent inflammasome activation promotes atherosclerosis-brief report. Arterioscler. Thromb. Vasc. Biol. 39, 1645-1651 (2019).

26. Toldo, S. \& Abbate, A. The NLRP3 inflammasome in acute myocardial infarction. Nat. Rev Cardiol. 15, 203-214 (2018).

27. Baldrighi, M., Mallat, Z. \& Li, X. NLRP3 inflammasome pathways in atherosclerosis. Atherosclerosis 267, 127-138 (2017).

28. Ackers-Johnson, M. et al. A simplified, Langendorff-free method for concomitant isolation of viable cardiac myocytes and nonmyocytes from the adult mouse heart. Circ. Res. 119, 909-920 (2016).

29. Kuhns, S. et al. The microtubule affinity regulating kinase MARK 4 promotes axoneme extension during early ciliogenesis. J. Cell Biol. 200, 505-522 (2013).

30. Szyk, A., Deaconescu, A. M., Piszczek, G. \& Roll-Mecak, A. Tubulin tyrosine ligase structure reveals adaptation of an ancient fold to bind and modify tubulin. Nat. Struct. Mol. Biol. 18, 1250-1258 (2011).

31. Arce, C. A. \& Barra, H. S. Release of C-terminal tyrosine from tubulin and microtubules at steady state. Biochem. J. 226, 311-317 (1985).

32. Kellogg, E. H. et al. Near-atomic model of microtubule-tau interactions. Science $\mathbf{3 6 0}$ 1242-1246 (2018).

33. Shigematsu, H. et al. Structural insight into microtubule stabilization and kinesin inhibition by tau family MAPs. J. Cell Biol. 217, 4155-4163 (2018).

34. Bottanelli, F. et al. Two-colour live-cell nanoscale imaging of intracellular targets. Nat. Commun. 7, 10778 (2016).

35. Barisic, M. et al. Microtubule detyrosination guides chromosomes during mitosis. Science 348, 799-803 (2015)

Publisher's note Springer Nature remains neutral with regard to jurisdictional claims in published maps and institutional affiliations.

(c) The Author(s), under exclusive licence to Springer Nature Limited 2021 


\section{Article}

\section{Methods}

\section{Data reporting}

The experiments were randomized when possible, and the investigators were blinded to allocation during experiments and outcome assessment when possible (further information on randomization and blinding is available in the Nature Research Reporting Summary linked to this Article.

\section{Mice}

All in vivo experiments using mice were approved by the UK Home Office and were performed under PPL PA4BDF775. All mice were on a C57BL/6 background and housed under standard temperature $\left(18-23^{\circ} \mathrm{C}\right)$ and humidity (40-60\%), with a 12-h light-dark cycle. Mark ${ }^{-/-}$mice were provided by Y. Shi ${ }^{24}$ and Mutant Mouse Resource and Research Center; Myh6- $\mathrm{mcm}^{+/}$Cre mice were originally from the Jackson Laboratory (Cre was expressed under mouse cardiac-specific $\alpha$-myosin heavy chain promoter ( $\alpha$-MHC; Myh6)); Mark $4^{f / f l}$ mice were from Taconic Biosciences. Myh6- $\mathrm{mcm}^{+-}$Cre mice were crossed with Mark $4^{f / f l}$ mice to generate Myh6- $\mathrm{mcm}^{+-} \mathrm{cre}$;Mark $4^{f l / f l}$ mice. The Cre-mediated excision of floxed Mark4 alleles was induced by treatment with tamoxifen dissolved in corn oil by intraperitoneal injection at $20 \mathrm{mg} \mathrm{kg}^{-1}$ body weight per day for 5 consecutive days.

\section{Left anterior descending coronary artery ligation model}

Permanent left anterior descending (LAD) coronary artery ligation was performed on mice as previously described ${ }^{22,23}$ with minor modifications. Mice, at $8-10$ weeks of age, were anaesthetized using ketamine at $100 \mathrm{mg} \mathrm{kg}^{-1}$ body weight and xylazine at $10 \mathrm{mg} \mathrm{kg}^{-1}$ body weight by intraperitoneal injection, and then intubated and ventilated with air (supplemented with oxygen) using a small-animal respirator. A thoracotomy was performed in the fourth left intercostal space. The left ventricle was visualized and the pericardial sac was ruptured to expose the LAD coronary artery. The LAD was permanently ligated using a 7-0 Prolene suture. The suture was passed approximately $2 \mathrm{~mm}$ below the tip of the left auricle. Marked colour changes of the ischaemic area and ECG changes were monitored as an indication of successful coronary artery occlusion. The thoracotomy was closed with 6-0 Prolene sutures. Sham-operated mice underwent the same procedure without coronary artery ligation. The endotracheal tube was removed once spontaneous respiration resumed, and the mice were placed in a warm recovery cage maintained at $37^{\circ} \mathrm{C}$ until they were completely awake. At the indicated time points in the experimental timeline, the mice were euthanized by $\mathrm{CO}_{2}$ asphyxiation and the tissues were subsequently collected for analysis.

\section{Bone marrow transplants}

Eight-to-ten-week-old C57BL/6 mice were maintained overnight with Baytril (Bayer) before irradiation with two doses of $5.5 \mathrm{~Gy}$ (separated by $4 \mathrm{~h}$ ) followed by reconstitution with $1 \times 10^{7}$ sex-matched donor bone marrow cells. Mice were randomly assigned to receive the $\mathrm{Mark4}^{-/-}$ or $\mathrm{Mark}^{+/+}$bone marrow. Mice were then maintained on Baytril for a 4-week recovery period before performing LAD ligation.

\section{Echocardiography}

Transthoracic echocardiography was performed on all mice using Vevo 3100 with a MX400 linear array transducer (VisualSonics), $30 \mathrm{MHz}$. Mice were anaesthetized with 2-3\% isoflurane and kept warm on a heated platform $\left(37^{\circ} \mathrm{C}\right)$. The chest hairs were removed using depilatory cream and a layer of acoustic coupling gel was applied to the thorax. After alignment in the transverse B-mode with the papillary muscles, cardiac function was measured on M-mode images. Echocardiography data were collected using VisualSonics Vevo 3100 and analysed using Vevo LAB3.1.1.

\section{Histological analysis}

Whole hearts were excised at different time point after LAD ligation, rinsed in PBS and fixed with $4 \%$ PFA overnight at $4{ }^{\circ} \mathrm{C}$. Fixed tissues were thoroughly washed in PBS, and then immersed in 30\% sucrose. Tissues were embedded and sectioned by a cryostat into $10-\mu \mathrm{m}$ thick slices, which started at the apex and ended at the suture ligation site. Masson's trichrome staining was performed to determine scar size. Scar size was calculated as the total infarct circumference divided by the total left ventricle circumference. Some hearts were excised at $24 \mathrm{~h}$ after myocardial infarction and quickly sliced into four $1.0-\mathrm{mm}$ thick sections perpendicular to the long axis of the heart. The sections were then incubated with $1 \%$ triphenyltetrazolium chloride (TTC, Sigma) for $15 \mathrm{~min}$ at $37^{\circ} \mathrm{C}$ and digitally photographed. To analyse the infarct size at $24 \mathrm{~h}$ after myocardial infarction, the TTC-stained area and TTC-negative area (infarcted myocardium) were measured using ImageJ (v.2.0). Myocardial infarct size was expressed as a percentage of the total left ventricle area. Images were obtained using a Leica DM6000 B microscope, collected using LAS AF software (2.4.0 build 6254) and analysed using ImageJ (v.2.0).

\section{Tissue immunohistochemistry}

Whole hearts were excised, quickly washed in PBS and flash-frozen. Tissues were then embedded and cryo-sectioned. Slices were fixed in pre-chilled methanol for $10 \mathrm{~min}$ at $-20^{\circ} \mathrm{C}$. After washing with PBST (0.1\% Tween-20 in $1 \times$ PBS), slices were incubated with $3 \% \mathrm{H}_{2} \mathrm{O}_{2}$ (in PBS) for $10 \mathrm{~min}$, and then with blocking buffer (5\% BSA in PBST) for $1 \mathrm{~h}$ at room temperature. The primary antibody against MARK4 (Abcam, ab124267, used at 1:200) or rabbit IgG isotype control (Novus Biologicals, NB81056910 , used at 1:1,000) was used overnight at $4{ }^{\circ} \mathrm{C}$. Extensive washing steps were performed to remove nonspecific antibody binding. Slices were incubated with the biotinylated secondary antibody (Abcam, ab6720, used at 1:800) for $1 \mathrm{~h}$ at room temperature. Reagents $A$ and $B$ from the Avidin-Biotin Complex kit (VECTOR, PK-4000) were diluted and added to the slides. The slides were stained with ImmPACT DAB peroxidase substrate (VECTOR, SK-4105) and counterstained with haematoxylin. Images were obtained using a Leica DM6000 B microscope, collected using LAS AF software (2.4.0 build 6254) and analysed using ImageJ (v.2.0) analyse tools.

\section{Microtubule co-sedimentation assay}

Lyophilized porcine brain tubulin (T240) was purchased from Cytoskeleton. Recombinant proteins of 4R-MAP4 and VASH2-SVBP were previously described ${ }^{12,33}$. Desiccated tubulin was reconstituted in the microtubule polymerization buffer to $10 \mathrm{mg} \mathrm{ml}^{-1}$. To generate polymerized microtubules, tubulin was diluted to $2 \mathrm{mg} \mathrm{ml}^{-1}$ in the polymerization buffer ( $80 \mathrm{mM} \mathrm{K}$-PIPES, pH 6.8, $1 \mathrm{mM} \mathrm{MgCl} 2,1 \mathrm{mMEGTA}$ and $1 \mathrm{mM}$ DTT), supplemented with $5 \%$ glycerol and $1 \mathrm{mM} \mathrm{GTP} \mathrm{at} 37^{\circ} \mathrm{C}$ for $30 \mathrm{~min}$, and then stabilized by incubating with $2.5 \mu \mathrm{M}$ taxol at $37^{\circ} \mathrm{C}$ for $15 \mathrm{~min}$. The taxol-stabilized microtubules were centrifuged over a cushion buffer (polymerization buffer with $40 \%$ glycerol) at $131,700 \mathrm{~g}$ at $37^{\circ} \mathrm{C}$ for $15 \mathrm{~min}$ to remove the free tubulin. The pellet was resuspended in the polymerization buffer with $1 \mu \mathrm{M}$ taxol. Taxol influenced the association of 4R-MAP4 with the microtubules in our assay. 4R-MAP4 association was facilitated when taxol was completely excluded from the buffer. The microtubules without taxol were susceptible to depolymerization if stored at room temperature. In these conditions, the polymerized microtubules were maintained at $37^{\circ} \mathrm{C}$ throughout the experiment. For the co-sedimentation assay, the microtubules were mixed with various concentrations of 4R-MAP4 $(1-6 \mu \mathrm{M})$ and VASH2-SVBP $(1-4 \mu \mathrm{M})$ in the polymerization buffer. In the competition experiments, the microtubules were incubated with specified 4R-MAP4 concentrations $(1-4 \mu \mathrm{M})$ for $10 \mathrm{~min}$, followed by addition of a constant amount of VASH2-SVBP $(3 \mu \mathrm{M})$ with further incubation for $10 \mathrm{~min}$. Subsequently, the reaction mixture was centrifuged using a TLA120.2 rotor at 55,000 rpm for 
$15 \mathrm{~min}$. The pellet fraction containing the microtubules and bound proteins was resuspended in the loading buffer. The samples were loaded on a $10 \%$ SDS-PAGE gel and stained with colloidal Coomassie blue dye (ThermoFisher). The experiments were repeated at least three times. The band intensities were analysed using ImageJ (v.2.0).

\section{Mouse cardiomyocyte isolation}

Preparation of cardiomyocytes was accomplished as previously described ${ }^{28}$. In brief, mice were anaesthetized and the chest was opened to expose the heart. the descending aorta was cut and the heart was immediately flushed by injection of $7 \mathrm{mlEDTA}$ buffer into the right ventricle. The ascending aorta was clamped and the heart was transferred to a $60-\mathrm{mm}$ dish containing fresh EDTA buffer. Digestion was achieved by sequential injection of $10 \mathrm{ml}$ EDTA buffer $(\mathrm{NaCl}, 130 \mathrm{mM} ; \mathrm{KCl}, 5 \mathrm{mM}$; $\mathrm{NaH}_{2} \mathrm{PO}_{4}, 0.5 \mathrm{mM}$; HEPES, $10 \mathrm{mM}$; glucose, $10 \mathrm{mM}$; BDM, $10 \mathrm{mM}$; taurine, $10 \mathrm{mM}$;EDTA, $5 \mathrm{mM}$; pH to 7.8), $3 \mathrm{ml}$ perfusion buffer $(\mathrm{NaCl}, 130 \mathrm{mM} ; \mathrm{KCl}$, $5 \mathrm{mM}$; $\mathrm{NaH}_{2} \mathrm{PO}_{4}, 0.5 \mathrm{mM}$; HEPES, $10 \mathrm{mM}$; glucose, $10 \mathrm{mM} ; \mathrm{BDM}, 10 \mathrm{mM}$; taurine, $10 \mathrm{mM} ; \mathrm{MgCl}_{2}, 1 \mathrm{mM} ; \mathrm{pH}$ to 7.8 ) and $30-50 \mathrm{ml}$ collagenase buffer (collagenase $2,0.5 \mathrm{mg} \mathrm{ml}^{-1}$; collagenase $4,0.5 \mathrm{~g} \mathrm{ml}^{-1}$; protease $\mathrm{XIV}, 0.05 \mathrm{mg} \mathrm{ml}^{-1}$; made fresh and diluted in perfusion buffer) into the left ventricle. The left ventricle was then separated and gently pulled into 1-mm pieces using forceps. Cellular dissociation was completed by gentle trituration, and enzyme activity was inhibited by addition of $5 \mathrm{ml}$ stop buffer (perfusion buffer containing $5 \%$ sterile FBS). The cell suspension was passed through a $100-\mu \mathrm{m}$ filter. Cells underwent four sequential rounds of gravity settling, using three intermediate calcium reintroduction buffers (buffer $1,75 \%$ perfusion buffer with $25 \%$ culture medium; buffer $2,50 \%$ perfusion buffer with $50 \%$ culture medium; buffer $3,25 \%$ perfusion buffer with $75 \%$ culture medium; culture medium comprised $0.1 \% \mathrm{BSA}, 1 \% \mathrm{ITS}, 10 \mathrm{mMBDM}, 1 \% \mathrm{CD}$ lipid and $5 \%$ penicillin-streptomycin in M199) to gradually restore the calcium concentration to physiological levels.

\section{Primary cardiomyocyte culture and adenoviral transduction}

Adenoviral vectors including pAdeno-SV40-GFP-Blank vector (Adv-null), pAdeno-Ttl-SV40-GFP vector (Adv-Ttl) (NM_027192.2) and pAdeno-Mark4-SV40-GFP vector (Adv-Mark4) (NM_172279.1) were purchased from Applied Biological Materials. Adenoviral vector pAV[shRNA]-EGFP-U6>mMap4 (Map4shRNA target sequence:AGAGTGGACTATCCGGATTAT), adenoviralvectorpAV[shRNA]-EGFP-U6>mVash2 (Vash2 shRNA target sequence: GAGAATCCTTGCCTATCAAAT) and adenoviral vector $\mathrm{pAV}$ [shRNA]-EGFP-U6 $>$ Scramble were purchased from VectorBuilder. Six-well plates or coverslips were coated with laminin at a final concentration of $5 \mathrm{\mu g} \mathrm{ml}^{-1}$ in PBS overnight at $4{ }^{\circ} \mathrm{C}$. The wells were washed and air-dried for $10 \mathrm{~min}$ before plating cells. After collecting the cells by gravity settling and calcium re-introduction, the final cardiomyocyte pellets were resuspended in $2 \mathrm{ml}$ culture medium and $2 \mathrm{ml}$ pre-equilibrated plating medium (0.1\% FBS, $10 \mathrm{mM}$ BDM and 5\% penicillin-streptomycin in M199) for culture. After incubation for $1 \mathrm{~h}$, the cell medium was changed with pre-equilibrated culture medium and adenovirus vectors were administered at $5 \times 10^{6} \mathrm{PFU} \mathrm{ml} \mathrm{m}^{-1}$. After co-culture with virus for $8 \mathrm{~h}$, fresh culture medium was used to wash and replace the old culture medium containing the virus. Cells were either used for the contractility assay and western blotting immediately after the medium change (in the experiments of overexpression of TTL), or collected at $48 \mathrm{~h}$ after transduction (in the experiments of Mark4 overexpression, Map4 shRNA and Vash2 shRNA) for the subsequent assays.

\section{Cardiomyocyte contractility assay}

Sarcomere shortening and relaxation were measured in freshly isolated left ventricular cardiomyocytes of mouse hearts using the integrated IonOptix contractility/photometry system. Cardiomyocytes were maintained in normal Tyrode's solution $\left(\mathrm{NaCl}, 140 \mathrm{mM} ; \mathrm{MgCl}_{2}, 0.5 \mathrm{mM}\right.$; $\mathrm{NaH}_{2} \mathrm{PO}_{4}, 0.33 \mathrm{mM}$; HEPES, $5 \mathrm{mM}$; glucose, $5.5 \mathrm{mM}$; $\mathrm{CaCl}_{2}, 1 \mathrm{mM} ; \mathrm{KCl}$, $5 \mathrm{mM} ; \mathrm{NaOH}, \mathrm{pH}$ to 7.4 ) at room temperature, electrically stimulated at $2 \mathrm{~Hz}$ using a field stimulator, and changes in sarcomere length were recorded. Basal and peak sarcomere length, maximum departure and return velocities and time to peak were measured. All measurements were performed at room temperature. For parthenolide (PTL) experiments, cardiomyocytes were treated with $10 \mu \mathrm{M} \mathrm{PTL}$ (Sigma P0667) or vehicle at room temperature in normal Tyrode's solution for $1 \mathrm{~h}$ before contractility measurements, and the vehicle-dimethyl sulfoxide (DMSO) - diluted in the same way was applied as control. All measurements were performed at room temperature within $4 \mathrm{~h}$. Data were collected and analysed using IonWizard 7.4.

\section{Calcium measurements}

Measurement of intracellular calcium was performed in freshly isolated left ventricular cardiomyocytes using the integrated IonOptix contractility/photometry system. Cardiomyocytes were loaded with $1 \mu \mathrm{M}$ Fura-2-AM for $20 \mathrm{~min}$ (protected from light) and then washed to allow de-esterification for $20 \mathrm{~min}$. Cells were then rinsed with normal Tyrode's solution. Cells were stimulated at $2 \mathrm{~Hz}$ using a field stimulator with dual excitation (at 360 and $380 \mathrm{~nm}$ ), and emission light was collected at $510 \mathrm{~nm}$. Changes in calcium transients were recorded using IonOptix software. All of the cells analysed were beating. All measurements were performed at room temperature within $4 \mathrm{~h}$. Data were collected and analysed using IonWizard 7.4.

\section{Immunofluorescence and image acquirement}

Cardiomyocytes were fixed with pre-chilled methanol for $10 \mathrm{~min}$, then washed twice using PBST ( $0.1 \%$ Tween-20 in $1 \times$ PBS) with 5-min intervals. Cells were blocked for $1 \mathrm{~h}$ at room temperature with blocking buffer (5\% BSA in PBST) and incubated with primary antibodies overnight at $4{ }^{\circ} \mathrm{C}$. The primary antibodies were: detyrosinated $\alpha$-tubulin (Abcam, ab48389, used at 1:200), $\alpha$-tubulin (CST,3873S, used at 1:200), MARK4 (Abcam, ab124267, used at 1:200), APC anti-mouse CD45 (BioLegend, 103112, used at 1:200) and rabbit IgG isotype control (Novus Biologicals, NB810-56910, used at 1:2,000). The cells were then washed with PBST and incubated with secondary antibody for $1 \mathrm{~h}$ at room temperature. The secondary antibodies were: AF488 donkey anti-rabbit IgG (Invitrogen, A21206, used at 1:200), AF647 goat anti-mouse IgG (Invitrogen, A21236, used at 1:200), AF647 goat anti-rat IgG (Invitrogen, A21247, used at 1:200). DAPI (Sigma, 10236276001, used at 1:1,000) was used. Confocal images were obtained using a Leica SP5 confocal laser scanning microscope, collected using LAS AF software (2.7.3.9723) and analysed using ImageJ (v.2.0) analyse tools.

\section{Stimulated emission depletion imaging and image analysis}

Cardiomyocytes on coverslips were fixed with $100 \%$ methanol for $15 \mathrm{~min}$ at room temperature and then washed three times with PBS (5-min intervals). Cells were blocked with buffer (5\% BSA and 0.2\% Triton X-100 in PBS) for $30 \mathrm{~min}$, then incubated with primary antibodies (diluted in blocking buffer) overnight at $4{ }^{\circ} \mathrm{C}$. The primary antibodies were VASH2 (Abcam, ab224723, used at 1:200), MAP4 (Abcam, ab245578, used at 1:200) and $\alpha$-tubulin (CST, 3873S, used at 1:200). The cells were washed three times using wash buffer (0.05\% Triton X-100 in PBS) at room temperature, then incubated with the secondary antibody for $1 \mathrm{~h}$ at room temperature. The secondary antibodies were: Atto-594 goat anti-rabbit IgG (Sigma, 77671, used at 1:500) and Atto-647N goat anti-mouse IgG (Sigma, 50185, used at 1:500). Cells were then washed three times in wash buffer. Cells were fixed ( $3 \%$ paraformaldehyde and $0.1 \%$ glutaraldehyde diluted in PBS) followed by three washes in PBS. The coverslips were then mounted on the slide.

Stimulated emission depletion (STED) imaging was carried out on a custom multicolour system with three pulsed excitation lines, one fixed depletion line, fast $16 \mathrm{kHz}$ beam scanning and gated detection centred around an Olympus IX83 microscope base. This system uses identical hardware, and a closely matched optical arrangement, to a previously described system ${ }^{34}$. In brief, two-colour STED imaging was performed 
sequentially. Images were acquired with a $100 \times$ oil-immersion objective lens (Olympus, UPLSAPO 100XO/PSF). Fields of view between 23 and $27 \mu \mathrm{m}^{2}$ were imaged with a 1,024 $\times 1,024$ image format and an approximately $20 \mathrm{~nm}$ pixel size. Excitation powers were between 15 and $30 \mu \mathrm{W}$ at the microscope side port and the STED depletion power was approximately $120 \mathrm{~mW}$ at the microscope side port. Fast, $16 \mathrm{kHz}$, unidirectional beam scanning with blanking was used to minimize light exposure. Each line of an image was scanned 850 times, resulting in an image acquisition time of approximately $54 \mathrm{~s}$ per colour. STED image data were collected with a custom program written in National Instrument (NI) LabVIEW 2014 64-bit, NI FPGA Module and NI Vision Development Module.

MAP4 oligomerized puncta (with a diameter longer than $400 \mathrm{~nm}$ ) were measured and calculated using ImageJ (v.2.0). The number of puncta was normalized against the cell area of each image.

For the acquired images, a dynamic thresholding algorithm was used for the image analysis. Images were converted into HSV colour images $(C)$ with information of hue $(h)$, saturation $(s)$ and value $(v) . C(i, j)$ was assumed as a non-background image pixel, $N$ was the total number of non-background image pixels, $H$ was image height and $W$ was image width. The average of all the non-background image pixels was calculated as $k=\left(\sum_{i=1}^{H} \sum_{j=1}^{W} C(i, j)\right) / N$. The following three thresholds were applied to discriminate signals: $h=[0,180] ; s=[0,43] ; v=[k+30,220]$. A Gaussian filter $\left(f(x)=\frac{1}{2 \pi \sigma^{2}} \mathrm{e}^{-\frac{x^{2}+y^{2}}{2 \sigma^{2}}}\right)$, a two-dimensional convolution operator, was used to remove noise. For the VASH2 signals, the Gaussian filter with a kernel of $3 \times 3$ was used for image denoising. For the linear microtubule signals, the Gaussian filter with a kernel of $5 \times 5$ was used for image denoising when $k \geq 35$ and a kernel of $3 \times 3$ was applied when $k<35$. The total numbers of VASH2 $(v)$ and $\alpha$-tubulin $(t)$ pixels were calculated. The total number of overlapping image pixels $(o)$ between VASH 2 and tubulin was calculated as VASH 2 signals on the microtubules, and $(1-o) / v$ was calculated as VASH 2 signals off the microtubules. The Pearson correlation coefficient $\left(\rho_{X, Y}=\frac{\operatorname{cov}(X, Y)}{\sigma_{X} \sigma_{Y}}\right)$ between VASH 2 signals and tubulin signals was calculated. Automatic image processing was coded using a custom algorithm in Python v.3.7.8.

\section{Subcellular fractionations of the primary cardiomyocytes}

Subcellular fractionations of primary cardiomyocytes were performed according to the manufacturer's instructions (Pierce, 87790). In brief, cells were incubated with $\mathrm{CEB}$, which selectively permeabilizes the cell membrane for 10 min at $4{ }^{\circ} \mathrm{C}$ with gentle mixing. Cells were centrifuged for $5 \mathrm{~min}$ at $500 \mathrm{~g}$ and supernatants were collected. The cytoskeletal binding proteins were isolated in the PEB.

Subcellular fractionations of primary cardiomyocytes were also obtained using a conventional method. Primary mouse cardiomyocytes were isolated and homogenized in pre-warmed $\left(37^{\circ} \mathrm{C}\right)$ microtubule-stabilizing buffer (PIPES, $80 \mathrm{mM} ; \mathrm{MgCl}_{2}, 1 \mathrm{mM}$; EGTA, $1 \mathrm{mM} ; 0.5 \%$ Triton X-100; $10 \%$ glycerol; GTP, $0.5 \mathrm{mM}$; Halt protease inhibitor cocktail from Thermo Fisher Scientific 1862209; $\mathrm{pH}$ to 6.8) using a Dounce homogenizer. The homogenates were centrifuged at $100,000 \mathrm{~g}$ for $15 \mathrm{~min}$ at room temperature. The supernatants were collected as the free-tubulin fraction as $\mathrm{F} 1$, and the pellets were dissolved in the microtubule-destabilizing buffer (Tris- $\mathrm{HCl}$, $20 \mathrm{mM}$; $\mathrm{NaCl}, 150 \mathrm{mM}$; 1\% Triton X-100; $\mathrm{CaCl}_{2}, 10 \mathrm{mM}$; Halt protease inhibitor cocktail from Thermo Fisher Scientific 1862209; $\mathrm{pH}$ to 7.4) for further incubation on ice for $1 \mathrm{~h}$ to depolymerize the microtubules. The dissolved lysates were centrifuged at $12,000 \mathrm{~g}$ for $15 \mathrm{~min}$ at $4{ }^{\circ} \mathrm{C}$. The pellets were incubated with 150 units of micrococcal nuclease (100 units $\mu^{-1}$, Thermo Fisher Scientific, 88216) in microtubule-destabilizing buffer for $15 \mathrm{~min}$ at room temperature, and then centrifuged at $12,000 \mathrm{~g}$ for $5 \mathrm{~min}$ at $4{ }^{\circ} \mathrm{C}$ to remove the nuclear debris. The collected pellets were dissolved in $2 \times$ SDS buffer (4\% SDS; $20 \%$ glycerol; Tris- $\mathrm{HCl}, 0.25 \mathrm{M}$; $\mathrm{pH}$ to 6.5 ). The dissolved lysates were then centrifuged at $14,000 \mathrm{~g}$ for $5 \mathrm{~min}$ at $4{ }^{\circ} \mathrm{C}$. The supernatants were collected as F2 (extraction from the stable pellet fraction) and the residual pellets were kept.

\section{Western blotting}

The heart tissues were grounded thoroughly with a mortar and pestled in liquid nitrogen. Tissue powder was lysed using Triton lysis buffer (20 mM Tris-HCl, pH to 7.5; $150 \mathrm{mM} \mathrm{NaCl} ; 1$ mM Na${ }_{2}$ EDTA; $1 \mathrm{mM}$ EGTA; $1 \%$ Triton X-100; $1 \mathrm{mM} \mathrm{Na} \mathrm{VO}_{4} ; 5 \mathrm{mM} \mathrm{NaF}$; protease inhibitor cocktail (ThermoFisher, 1862209)). The supernatant (soluble fraction) was collected, and the pellets (insoluble fraction) were dissolved in $8 \mathrm{M}$ urea (Figs. 1b, 3a, d; $n=12$ mice in the Mark $^{+/+}$myocardial infarction group and $n=9$ mice in the $\mathrm{Mark}^{-/-}$myocardial infarction group used for Fig. $3 \mathrm{~b}$ ). For some experiments ( $n=8$ mice per group used for Fig. 3b, c), heart tissues were homogenized in the lysis buffer (0.1 M PIPES pH to 6.8; 2 mM EGTA; 0.1 mM EDTA; $0.5 \mathrm{mM}$ $\mathrm{MgCl}_{2} ; 20 \%$ glycerol; $0.1 \%$ Triton X-100; protease inhibitor cocktail (ThermoFisher, 1862209)) and incubated for $30 \mathrm{~min}$ at $37^{\circ} \mathrm{C}$. After centrifugation $(21,100 \mathrm{~g}$ for $5 \mathrm{~min})$, the supernatants were collected as the soluble fraction, and the pellets were dissolved in buffer (RIPA buffer (CST, 9806); 0.8\% SDS; and protease inhibitor cocktail (ThermoFisher, 1862209)) and collected as the insoluble fraction. The protein concentration was determined using a BCA protein assay kit (ThermoFisher, 23235). Molecular mass markers (ThermoFisher, LC5603 and LC5925) were used. Supernatant samples were prepared in NuPAGE LDS sample buffer (Invitrogen) and run on NuPAGE 4-12\% Bis-Tris gels (Invitrogen). Pellet samples were prepared in Tris-glycine SDS sample buffer (Invitrogen) and run on Novex 4-20\% Tris-glycine gels (Invitrogen). All samples were blotted onto a PVDF membrane after electrophoresis. The following primary antibodies were used in the experiments: MARK4 (CST, 4834S, used at 1:1,000), GAPDH (CST, 5174S, used at 1:1,000), desmin (R\&D, AF3844, used at 1:1,000), detyrosinated $\alpha$-tubulin (Abcam, ab48389, used at 1:200), polyglutamylated $\alpha$-tubulin (AdipoGen, AG-20B-0020-C100, used at 1:1,000), acetylated $\alpha$-tubulin (Santa Cruz Biotechnology, sc23950, used at $1: 1,000)$, $\alpha$-tubulin (CST, 3873S, used at 1:200). After antibody detection, membranes were revealed with ECL. Quantification of the western blot bands was performed using ImageJ (v.2.0).

For the fractionation assay, equal amounts of total protein $(20 \mu \mathrm{g})$ from each fraction were used for western blot. The DC protein assay kit (Bio-Rad, 5000111) was used to measure protein concentrations. Across different gels, equal amounts of a molecular mass marker (ThermoFisher, LC5603) were loaded in each gel. Samples were run on NuPAGE 4-12\% Bis-Tris gels (Invitrogen) and blotted onto a PVDF membrane.

Some samples of the CEB fraction from the fractionation assay were prepared for native gel analysis. Samples were processed in Tris-glycine native sample buffer (ThermoFisher, LC2673) before loading without heating or addition of any reducing reagent. Samples were loaded on a 3-8\% NuPAGE Tris-acetate gel (ThermoFisher, EA0375BOX) for electrophoresis in Tris-glycine native running buffer (Tris-base, $25 \mathrm{mM}$; glycine, $192 \mathrm{mM}$; pH to 8.3). Native molecular marker (ThermoFisher, LC0725) was used. After electrophoresis, proteins were transferred to a PVDF membrane by transfer buffer (Bicine, 25mM; Bis-Tris, $25 \mathrm{mM}$; EDTA, $1 \mathrm{mM}$; pH to 7.2).

Some samples from the fractionation assay were prepared with a denaturing treatment by adding urea. Urea $(0 \mathrm{M}, 2 \mathrm{M}, 4 \mathrm{M}$ or $8 \mathrm{M})$ was added to the samples as indicated. A micro-BCA protein assay kit (ThermoFisher, 23235) was used to measure protein concentrations if urea was added to the samples. Samples were then processed in Tris-glycine SDS sample buffer (ThermoFisher, LC2676) and reducing reagent (10\% 2-mercaptoethanol). A 4-20\% Tris-glycine gel (ThermoFisher, EC6026BOX) was used for electrophoresis in Tris-glycine SDS running buffer (Tris-base, $25 \mathrm{mM}$; glycine, $192 \mathrm{mM}$; 0.1\% SDS; $\mathrm{pH}$ to 8.3). 
After electrophoresis, proteins were transferred to PVDF membrane by transfer buffer (Tris-base, $12 \mathrm{mM}$; glycine, $96 \mathrm{mM}$; $\mathrm{pH}$ to 8.3).

The primary antibodies used for fractionation assays were: detyrosinated $\alpha$-tubulin (Abcam, ab48389, used at 1:1,000), $\alpha$-tubulin (CST, 3873S, used at 1:1,000), TTL (Proteintech, 13618-1-AP, used at 1:1,000), VASH1 (Abcam, ab199732, used at 1:1,000), VASH2 (Abcam, ab224723, used at 1:1,000), pMAP4(S1073) (Abnova, PAB15916, used at 1:1,000), MAP4 (Abcam, ab245578, used at 1:1,000), pMAP4(S941) (Abcam, ab56087, used at 1:1,000), GAPDH (CST, 5174S, used at 1:1,000), desmin (R\&D, AF3844, used at 1:1,000). Membranes were revealed with ECL. Quantification of western blot bands was performed using ImageJ (v.2.0). The band density was normalized in two steps: (1) the density of the targeted band was first normalized against the density of the loading molecular mass marker band (norm 1); (2) the value of norm 1 was internally normalized against the average value of norm 1 of the control group (norm 2). The finalized value (norm 2) was used to compare the fold changes against the value of the control groups across different gels. desmin was used as a marker for the pellet fraction, and GAPDH was used as a marker for the cytosolic fraction. Coomassie-blue-stained gels loaded with the same amounts of proteins as used in western blotting experiments or Ponceau-S-stained membranes after the transfer step were used to confirm equal loading. All of the immunoblots, gels and membranes associated with the data presented in the figures and extended data figures are provided (Supplementary Fig. 1).

\section{Heart tissue digestion and flow cytometry}

Hearts were collected and the left ventricle was isolated, minced with fine scissors and subjected to enzymatic digestion solution (RPMI 1640 , collagenase $\mathrm{D}\left(0.2 \mathrm{mg} \mathrm{ml}^{-1}\right.$, Roche $)$, dispase $\left(1 \mathrm{U} \mathrm{ml}^{-1}\right.$, StemCell Technologies $)$ and DNase I $\left(0.2 \mathrm{mg} \mathrm{ml}^{-1}\right.$, Sigma $\left.)\right)$ for $45 \mathrm{~min}$ at $37^{\circ} \mathrm{C}$. Cells were collected, filtered through a $40-\mu$ m nylon mesh and washed with PBS with $2.5 \% \mathrm{v} / \mathrm{v}$ fetal bovine serum. Cell suspensions were incubated with a Zombie Aqua Fixable Viability Kit (Biolegend, 423102, used at $1: 1,000)$ for $20 \mathrm{~min}$ at room temperature and washed with PBS. Cells were then stained with fluorescently labelled anti-mouse antibodies, including APC anti-mouse CD45 (Biolegend, 103112, used at 1:100), AF488 anti-mouse CD11b (Biolegend, 101217, used at 1:100), Pacific blue anti-mouse Ly6G (Biolegend, 127612, used at 1:100), PE anti-mouse F4/80 (Biolegend, 123110, used at 1:100), PECY7 anti-mouse CD11C (Biolegend, 117318, used at 1:100), Brilliant Violet 605 anti-mouse CD3 (Biolegend, 100237, used at 1:100) and FITC anti-mouse CD19 (Biolegend, 553785, used at 1:100), diluted in staining buffer for 30 min at $4{ }^{\circ} \mathrm{C}$ in the presence of $24 \mathrm{G} 2 \mathrm{Fc}$ receptor blocker (obtained from the Division of Immunology, Department of Pathology, University of Cambridge), before extensive washing. The cytometric acquisition was performed on a LSR II Fortessa (BD biosciences). Cell analysis was done using BD FACSDiva Software 6.0 and FlowJo software (v.10).

\section{Real-time PCR}

For gene expression analysis, RNA from heart tissues or separated cardiomyocytes was isolated using an RNAeasy mini kit (Qiagen). Reverse transcription was performed using a QuantiTect reverse-transcription kit (Qiagen). qRT-PCR was performed with SYBR Green qPCR mix (Eurogentec) using the Roche LightCycler 480II. Primer sequences are as follows: Mark4 (forward 5'-GGACACGCATGGCACATTG-3'; reverse 5'-GCAGGAAGCGATAGAGTTCCG-3'); Vash2 (forward 5'-GCC TTCCTGGCTAAGCCTTC-3'; reverse 5'-CCCTGTGTGGTTGTATTGT AGAG-3'); Hprt (forward 5'-TCAGTCAACGGGGGACATAAA-3'; reverse $5^{\prime}$-GGGGCTGTACTGCTTAACCAG-3'); Rpl4 (forward 5'-CCGT CCCСTCATATCGGTGTA-3'; reverse 5'-GCATAGGGCTGTCTGTT GTTTTT-3'); Rpl13a (forward 5'-AGCCTACCAGAAAGTTTGCTTAC-3'; reverse $5^{\prime}$-GCTTCTTCTTCCGATAGTGCATC- $\left.3^{\prime}\right)$. The average of three housekeeping genes ( $H p r t, R p l 4$ and Rpl13a) was used as reference for qPCR gene expression analysis.
Measurement of cardiac troponin I and inflammatory cytokines Serum was collected within $24 \mathrm{~h}$ after myocardial infarction or at day 3 after myocardial infarction. Measurements of the cardiac injury biomarker (collected within $24 \mathrm{~h}$ ) and cytokines (collected at day 3 after myocardial infarction) were performed by the core biochemical assay laboratory of Cambridge University Hospitals.

\section{Statistics and reproducibility}

All values in the text and figures are presented as mean \pm s.e.m. of independent experiments with given $n$ sizes. No statistical methods were used to predetermine sample size. Statistical analysis was performed with Prism v.7.05 (GraphPad) and Excel (Microsoft Excel 2102). Violin plots were created with Prism v.9.1.0 (216) (GraphPad). Data were tested for normality using a Kolmogorov-Smirnov test. Group comparisons were analysed using two-tailed analyses. Comparisons of three or more groups were analysed using one-way (one variable) or two-way (two variables) ANOVA followed by the Bonferroni post hoc correction for multiple comparisons when appropriate. $P<0.05$ was considered statistically significant.

Data in Figs. 1b, c, 3a and Extended Data Figs. 2a, 5a, 7b, 8a, b are representative of three independent experiments. Data in Extended Data Fig. $8 \mathrm{e}-\mathrm{g}$ are representative of two independent experiments.

\section{Reporting summary}

Further information on research design is available in the Nature Research Reporting Summary linked to this paper.

\section{Data availability}

All of the associated raw data presented in this paper are available from the corresponding author upon request. Source data are provided with this paper.

\section{Code availability}

Custom STED microscope control software used for data collection can be accessed at https://github.com/Gurdon-Super-Res-Lab/ STED-Control, and a customized algorithm used for STED image analysis is available at https://github.com/zhaoaite/dynamic_thresholding_algorithm.

Acknowledgements The work is supported by a British Heart Foundation (BHF) fellowship grant (FS/14/28/30713) to X.L., an Isaac Newton Trust grant (18.40u) to X.L. and Cambridge BHF Centre of Research Excellence grants (RE/13/6/30180 and RE/18/1/34212) to X.L. X.Y. was supported by a Royal Society Newton Advanced Fellowship grant (NA140277) and is supported by $\mathrm{BHF}$ grants (FS/14/28/30713, RE/18/1/34212). Z.M. is supported by $\mathrm{BHF}$ chair grant $(\mathrm{CH} / 10 / 001 / 27642)$ and the NIHR Cambridge Biomedical Research Centre. M.A.-N. is supported by German Research Foundation (Deutsche Forschungsgemeinschaft (DFG)) (AM 507/1-1). G.S., E.A. and D.S.J. are supported by a Wellcome Trust collaborative award (203285), a Wellcome Principal Research Fellowship (207496) and core support from the Wellcome Trust (203144) and Cancer Research UK (A24823). The work of H.Z. is supported in part by a Royal Society Newton Advanced Fellowship grant (NA160342). We thank staff at the phenotyping hub and biochemical assay laboratory of Cambridge University Hospitals; staff at the Phenomics Laboratory and Anne McLaren Building of Cambridge University; B. Prosser for sharing protocols and discussion; M. Zhang for advice on echography data; M. A. Ackers-Johnson from R. Foo's laboratory for technical advice on isolating and culturing primary mouse cardiomyocytes; S. Ozanne and L. Pantaleao for access and training of ultracentrifuge use; D. Pavlovic for advice on lonOptix; C. Huang for advice on calcium measurements; T. Zhao for his intellectual discussion and reading the manuscript; and X. Luo, who passed away due to COVID-19 during the outbreak in Wuhan, for her support of this project.

Author contributions $X, Y, X, C, M . A,-N, A . Z, H . Z$ and $X . L$ designed the experiments. $X, Y, X . C$ M.A.-N., E.A., A.Z., H.C., M.C., J.H. and X.L. performed the experiments. X.Y., X.C., M.A.-N., H.C., A.Z., H.Z. and X.L. analysed the data. X.Y. performed all of the in vivo experiments using mouse models and contractility assays, and wrote the relevant Methods section. X.C. performed the primary cardiomyocyte isolation, fractionation, real-time PCR, western blotting and imaging experiments. M.A.-N. performed in vitro microtubule co-sedimentation assay, analysed the data and wrote the relevant Methods section. E.A. configured the custom STED system, performed STED imaging and wrote the relevant Methods section. G.S. provided assistance for STED imaging. A.Z. and H.Z. wrote the STED image analytic code, analysed STED data and wrote the 


\section{Article}

relevant Methods section. H.C. assisted with tissue collection, performed tissue sectioning and staining assays, and optimized some of the experimental conditions. M.C. assisted with part of the tissue collection, staining and analysis. J.H. provided some technical supports on mouse experiments. C.D., H.C. and X.L. performed the initial test. H.H. and K.T. provided recombinant proteins. T.K. provided initial training for the LAD model. D.S.J. provided the super-resolution imaging platform. Z.M. independently had the idea and supported the initiation of the project (the mouse in vivo work was performed under the Procedure Project Licence of Z.M.). X.Y., X.C. Z.M. and X.L. interpreted the data for important intellectual contents. X.L. conceived idea, designed and initiated the project, established the collaboration, supervised the project, and wrote the manuscript. All authors reviewed and edited the manuscript.
Competing interests The authors declare no competing interests.

Additional information

Supplementary information The online version contains supplementary material available at https://doi.org/10.1038/s41586-021-03573-5.

Correspondence and requests for materials should be addressed to X.L.

Peer review information Nature thanks Leslie Leinwand and the other, anonymous, reviewer(s) for their contribution to the peer review of this work.

Reprints and permissions information is available at http://www.nature.com/reprints 
a

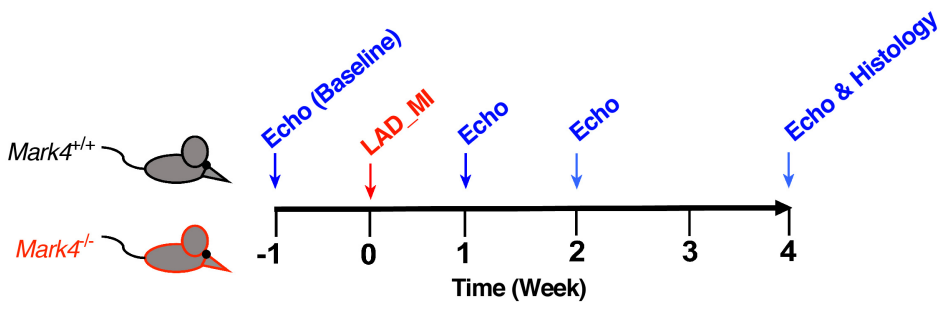

b

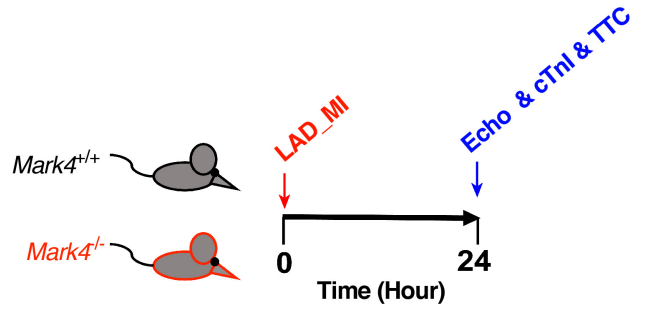

C

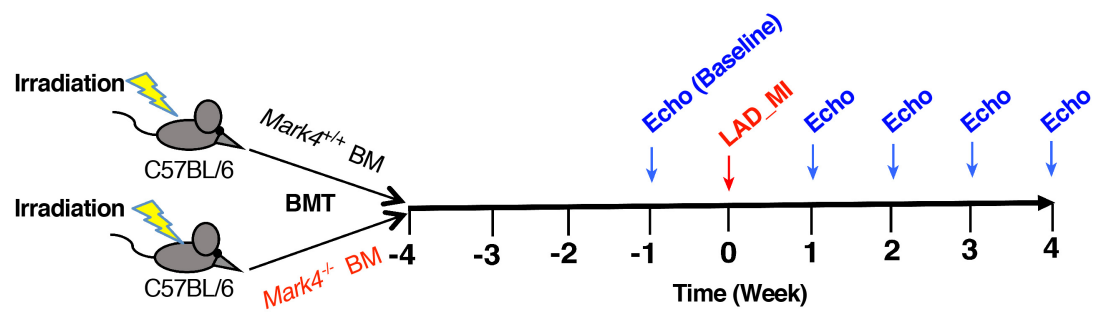

d
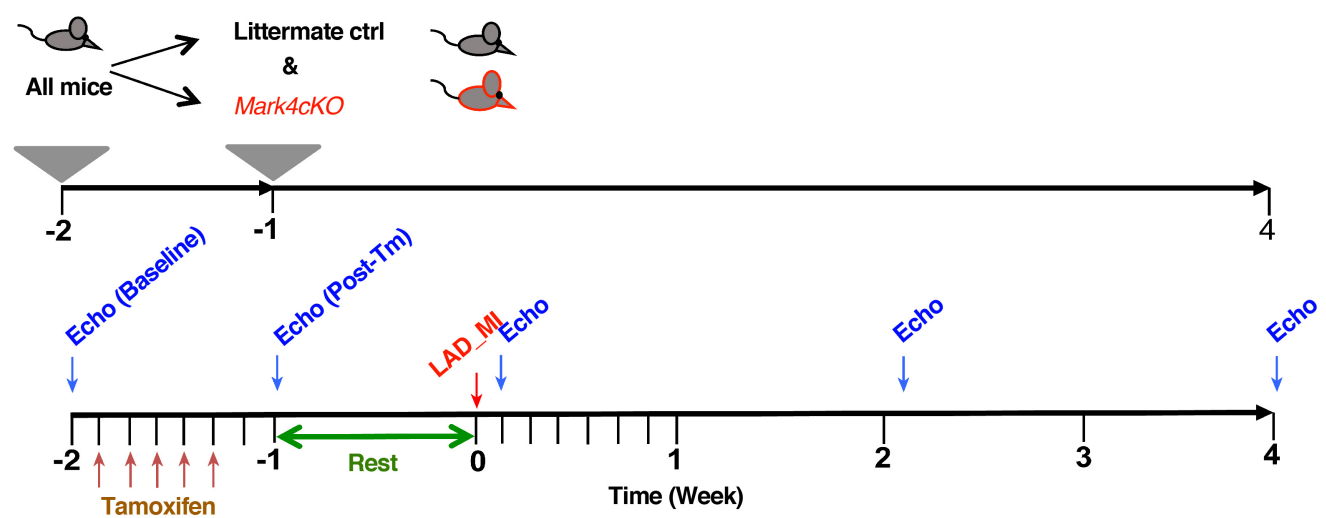

Extended Data Fig. $1 \mid$ Timeline of experimental design. a, Timeline of the experimental design for Fig.1d, e. Investigation of the effect of total MARK4 deficiency on cardiac function using the model of LAD coronary artery ligation to induce myocardial infarction. Echocardiography (Echo) and histological analyses were performed at the indicated time points. b. Timeline of the experimental design for Fig. 2a-c. Investigation of the effect of total MARK4 deficiency on cardiac function at $24 \mathrm{~h}$ after myocardial infarction. Echocardiography, circulating cardiac troponin and histological analyses were performed at the indicated time point. c, Timeline of the experimental design for Fig. 2d and Extended Data Fig. 3a, b. Investigation of the effect of MARK4 expression in haematopoietic cells on cardiac function using the LAD ligation model. BM, bone marrow; BMT, bone marrow transplantation. Echocardiography analysis was performed at the indicated time points. d, Timeline of the experimental design for Fig. 2e and Extended Data Fig. 3c-e. Investigation of the effect of MARK4 expression in cardiomyocytes on cardiac function using the LAD ligation model. Tm, tamoxifen. Mark $4 \mathrm{cKO}$, Mark 4 conditional knockout mice. Echocardiography analysis was performed at the indicated time points. 


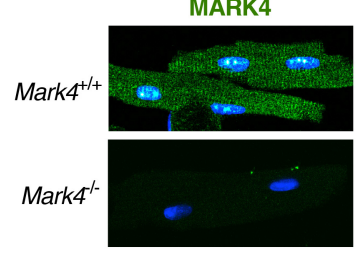

WT: Baseline

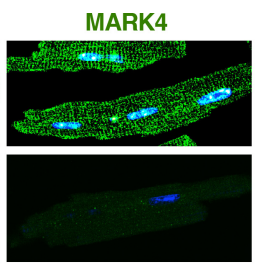

WT: D3 post-MI
Isotype ctrl

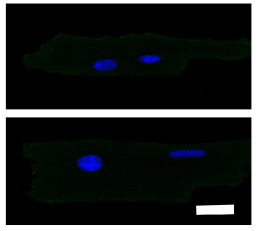

WT: D3 post-MI
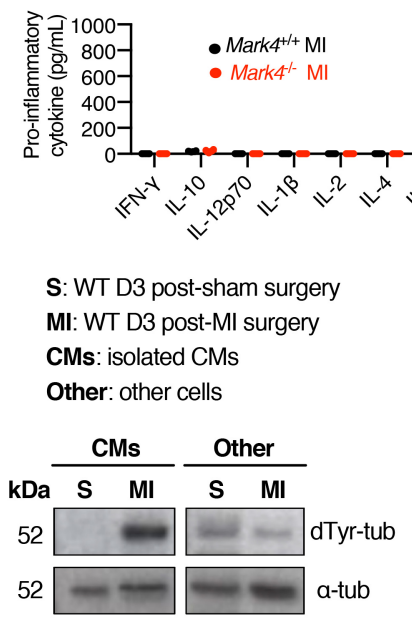

f

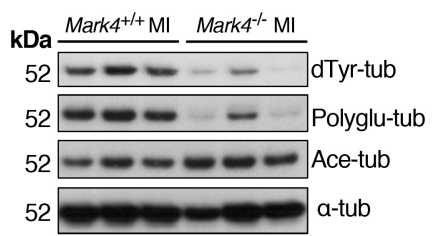

Extended Data Fig. 2 | MARK4 expression, $\alpha$-tubulin posttranslational modifications and changes in the inflammatory response after myocardial infarction. a, Representative confocal images of primary cardiomyocytes (CMs) isolated from $\mathrm{Mark4}^{-/}$or control mice at baseline or at day 3 after myocardial infarction. Scale bar, $20 \mu \mathrm{m}$. b, Levels of pro-inflammatory cytokines at day 3 after myocardial infarction ( $n=6$ per group). c, LVEF at day 3 after myocardial infarction ( $n=4$ per group). d, e, Western blots of detyrosinated $\alpha$-tubulin in cell lysates of cardiomyocytes isolated from wild-type mice at day 3 after myocardial infarction or post-sham surgery (S), with the lysates of the remaining cells from the same hearts used as control. d, Representative western blots. e, Ratio of detyrosinated $\alpha$-tubulin over total $\alpha$-tubulin quantified using western blot data from biologically independent c

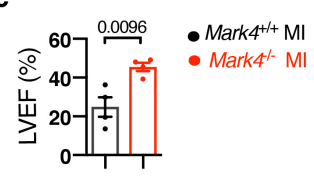

g $\quad \mathrm{Mark}^{+/+} \mathrm{MI}$

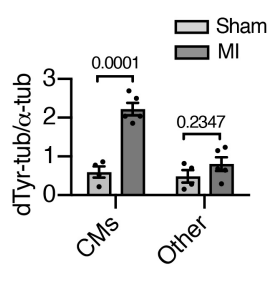

- Mark4t- Ml

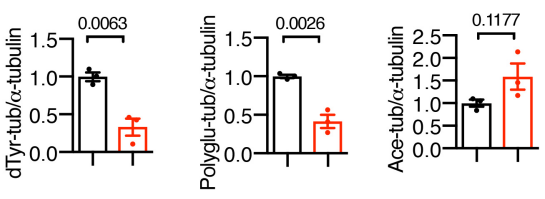

samples. Sham group, $n=4$ mice; myocardial infarction group, $n=5$ mice.

$\mathbf{f}, \mathbf{g}$, Western blots of cell lysates from the isolated cardiomyocytes of $\mathrm{Mark4}^{-/-}$ or control mice at day 3 after myocardial infarction to detect detyrosinated $\alpha$-tubulin, polyglutamylated $\alpha$-tubulin (Polyglu-tub), acetylated $\alpha$-tubulin (Ace-tub) and $\alpha$-tubulin.f, Representative western blots. g, Ratio of detyrosinated $\alpha$-tubulin, polyglutamylated $\alpha$-tubulin or acetylated $\alpha$-tubulin over total $\alpha$-tubulin quantified using western blot data from biologically independent samples ( $n=3$ mice per group). b. The box bounds represent the 25th and 75th percentiles, the middle line shows the median, the whiskers show the minimum and maximum, and individual replicates are shown as circles. $\mathbf{c}, \mathbf{e}, \mathbf{g}$, Data are mean \pm s.e.m. b, c, e, $\mathbf{g}$, Two-tailed unpaired $t$-tests were used; $P$ values are indicated. 
MARK4

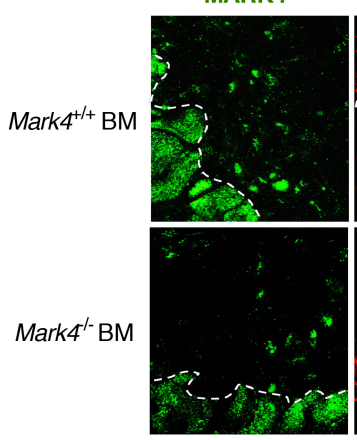

b

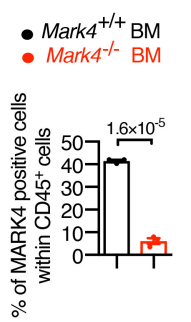

d

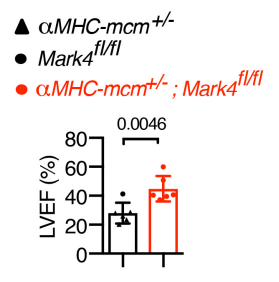

Extended Data Fig. 3 | Validation of the mouse models for MARK4 selective expression in either haematopoietic cells or cardiomyocytes.

a, b, Confirmation of MARK4 deficiency in $\mathrm{CD} 45^{+}$cells of chimeric wild-type mice reconstituted with bone marrow (BM) cells from $\mathrm{Mark4}^{-/-}$mice (strategy is shown in Extended Data Fig. 1c). a, Representative image with arrows pointing to $\mathrm{CD} 45^{+}$cells in the infarct area. Scale bars, $20 \mu \mathrm{m}$ (a). b, Quantification of the percentage of MARK $4^{+}$cells (green) within $C D 45^{+}$cells (red). $n=3$ mice per group. c, Confirmation of Mark4 deletion in cardiomyocytes (strategy is shown

C

e
CD45
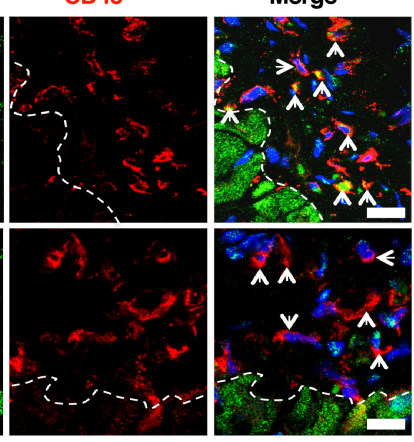

$\Delta \mathrm{MMHC}_{\mathrm{M}} \mathrm{mcm}^{+/-}$
- $\mathrm{Mark4}^{\text {fl/fI }}$

- $\alpha M H C-\mathrm{mcm}^{+/-} ;$Mark4 $^{\text {fl/fl }}$
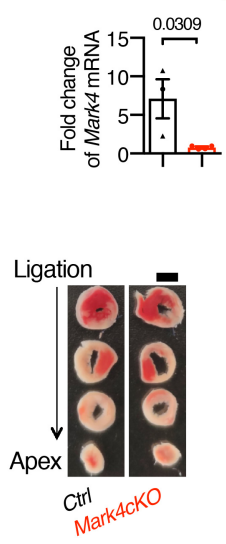

$\Delta \mathrm{\alpha MHC}-\mathrm{mcm}^{+/}$

- Mark4 ${ }^{f l / f I}$

- aMHC-mcm $^{+/}$; Mark4 $4^{f l f f l}$

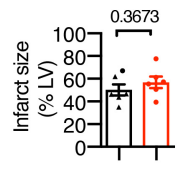

in Extended Data Fig. 1d). Real-time PCR of Mark4 level in primary cardiomyocytes isolated from $M y h 6-\mathrm{mcm}^{+/-} ; M^{\prime}$ ark $4^{f l / f l}$ (also known as

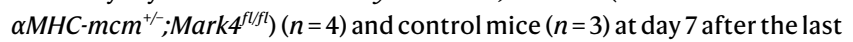
tamoxifen injection. d, Assessment of LVEF of a different group (compared with Fig. $2 \mathrm{e}$ ) of Mark $4 \mathrm{cKO}$ and control mice ( $n=6$ per group) at day 1 after myocardial infarction. e, Infarct size at $24 \mathrm{~h}$ after myocardial infarction ( $n=6$ per group). Scale bar, $2 \mathrm{~mm}$. b-e, Data are mean \pm s.e.m.; two-tailed unpaired $t$-test; $P$ values are indicated. 
a

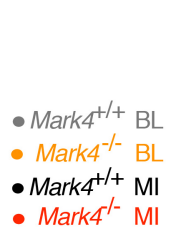

b

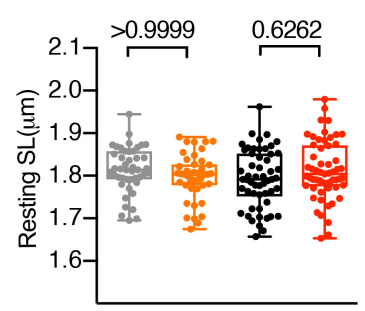

C

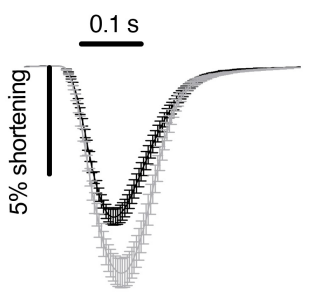

d

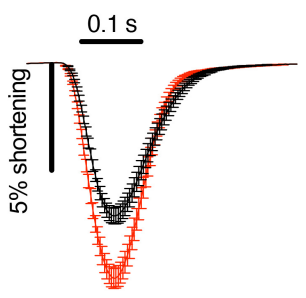

e

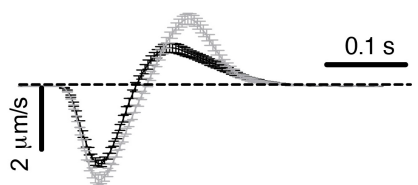

$\mathbf{f}$

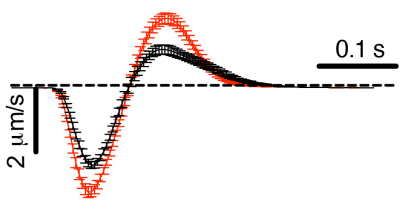

i

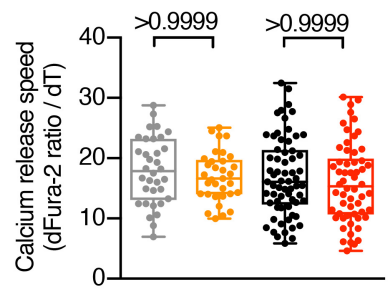

I

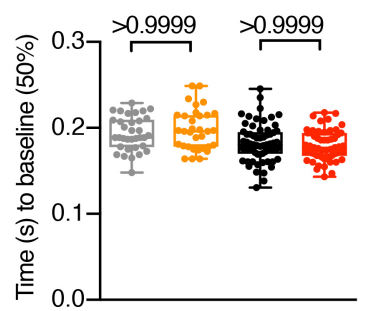

Extended Data Fig. 4 | The effect of MARK4 deficiency on sarcomere length, peak shortening, velocity and calcium transients in cardiomyocytes before and after myocardial infarction. a-f, Contractility assay of single primary cardiomyocytes isolated at baseline or at day 3 after myocardial infarction from the following groups: Mark $^{+/+}$baseline $(n=4$ mice, $n=45$ cardiomyocytes examined over 4 independent experiments), Mark4 $^{-/-}$ baseline ( $n=3$ mice, $n=45$ cardiomyocytes examined over 3 independent experiments), Mark $4^{+/+}$myocardial infarction ( $n=5$ mice, $n=54$ cardiomyocytes examined over 5 independent experiments) and Mark $^{-/-}$myocardial infarction ( $n=6$ mice, $n=57$ cardiomyocytes examined over 6 independent experiments). a, Colour denotation of samples. b, Resting sarcomere length. c, d, Average sarcomere shortening traces were compared.e,f, Average velocity traces $(\mathrm{dSL} / \mathrm{dT}) . \mathbf{g}-\mathbf{m}$, Calcium influx assay on single cardiomyocytes isolated from $\mathrm{Mark}^{-/-}$or control mice at baseline or at day 3 after myocardial infarction in the

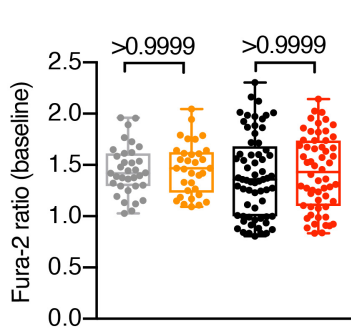

h

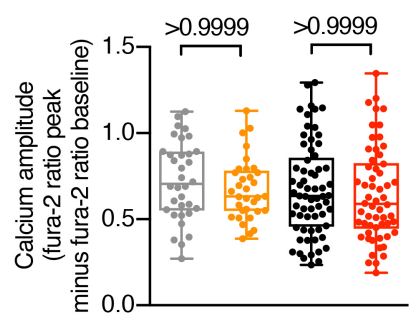

$\mathbf{k}$

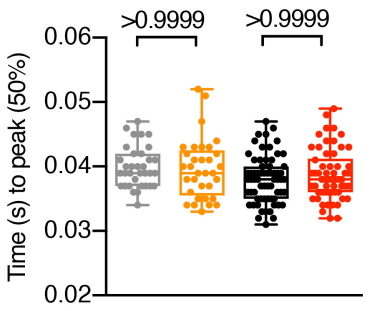

following groups: $\operatorname{Mark}^{+/+}$baseline group $(n=2$ mice, $n=34$ cardiomyocytes examined over 2 independent experiments), $\operatorname{Mark4}^{-/-}$baseline group ( $n=2$ mice, $n=33$ cardiomyocytes examined over 2 independent experiments), Mark $^{+/+}$myocardial infarction group $(n=4$ mice, $n=65$ cardiomyocytes examined over 4 independent experiments) and Mark $^{-/-}$myocardial infarction group ( $n=3$ mice, $n=58$ cardiomyocytes examined over 3 independent experiments).g, Basal $\mathrm{Ca}^{2+}$ level. h, Amplitude level of $\mathrm{Ca}^{2+}$ transients. $\mathbf{i}, \mathrm{Ca}^{2+}$ release speed during contraction. $\mathbf{j}, \mathrm{Ca}^{2+}$ reuptake speed during contraction. $\mathbf{k}, \mathrm{Ca}^{2+}$ elevation time. $\mathbf{l}, \mathrm{Ca}^{2+}$ reuptake time. $\mathbf{m}$, Traces of $\mathrm{Ca}^{2+}$ kinetic curves. b, g-l, The box bounds represent the 25 th and 75 th percentiles, the middle line shows the median, the whiskers show the minimum and maximum, and individual cardiomyocytes are shown as circles. b, g-l, Two-way ANOVA with Bonferroni post hoc correction for multiple comparisons; $P$ values are indicated. 
a

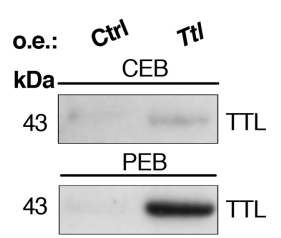

d

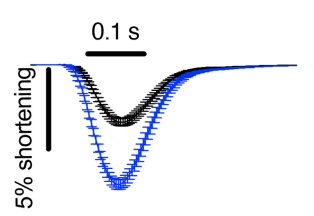

g

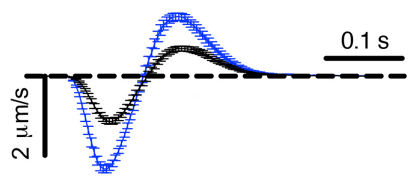

j

- Mark4 $^{+/+} \mathrm{MI}$ : DMSO

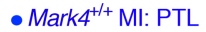

- Mark4 $4^{--} \mathrm{MI}$ : DMSO

- Mark4 ${ }^{-}$MI: PTL
- $\mathrm{Mark}^{+/+} \mathrm{Ml}$ : Adv-null o.e

- Mark4+/+ Ml: Adv-TTL o.e.

- Mark4-1-Ml: Adv-null o.e.

- Mark4t- MI: Adv-TTL o.e.

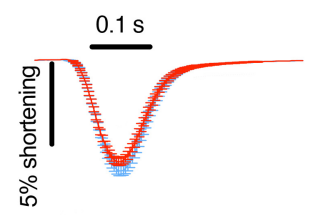

h

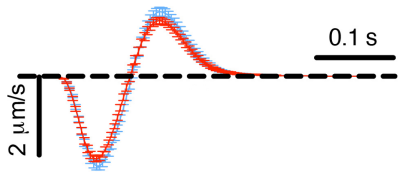

k

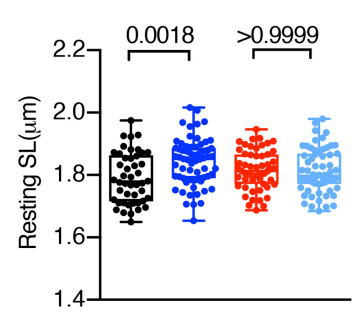

c
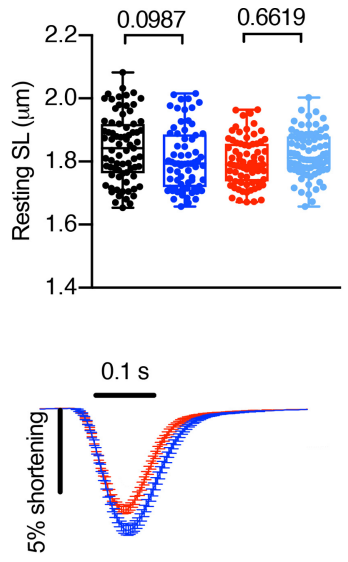

m

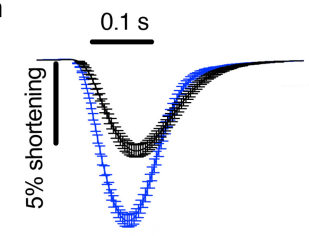

n

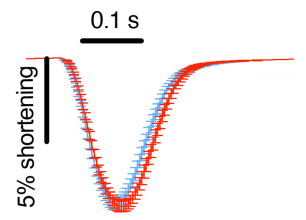

o

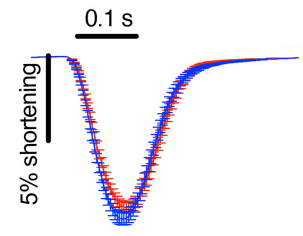

p

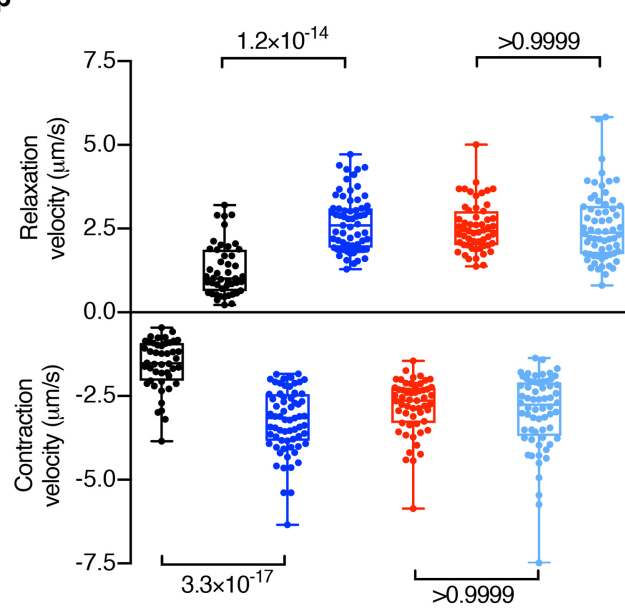

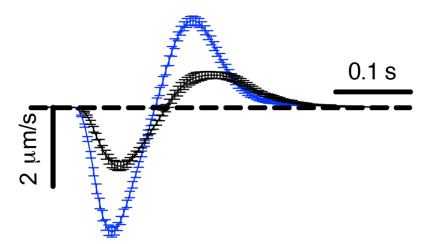

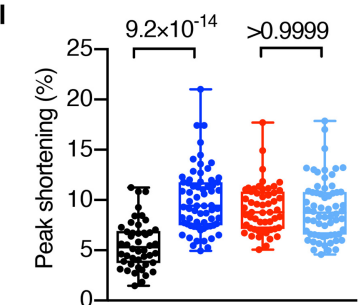




\section{Article}

Extended Data Fig. 5 | The effect of TTL overexpression, or PTL treatment, on contractility of $\mathrm{Mark4}^{-/-}$cardiomyocytes after myocardial infarction.

a-i, Adenovirus (Adv)-mediated overexpression (o.e.) of TTL in

cardiomyocytes isolated from $\mathrm{Mark}^{-/-}$or control $\mathrm{Mark}^{+/+}$mice at day 3 after myocardial infarction, with overexpression of a null vector as control (Ctrl). a, Representative western blot. b-i, Contractility assay of single cardiomyocytes with overexpression in the following groups: Mark4 $^{+/+}$ myocardial infarction Adv-Null ( $n=3$ mice, $n=75$ cardiomyocytes examined over 3 independent experiments), Mark4 $^{+/+}$myocardial infarction Adv-TTL ( $n=3$ mice, $n=69$ cardiomyocytes examined over 3 independent experiments), Mark $^{-/-}$myocardial infarction Adv-Null ( $n=3$ mice, $n=74$ cardiomyocytes examined over 3 independent experiments) and Mark $^{-/-}$myocardial infarction $\operatorname{Adv}$-TTL ( $n=3$ mice, $n=73$ cardiomyocytes examined over 3 independent experiments). b, Colour denotation of samples. c, Resting sarcomere length. d-f, Average sarcomere shortening traces. $\mathbf{g}-\mathbf{i}$, Average velocity traces $(\mathrm{dSL} / \mathrm{dT}) . \mathbf{j}-\mathbf{s}$, Contractility assay of single cardiomyocytes isolated at day 3 after myocardial infarction with the following treatments: ark $^{+/+}$myocardial infarction DMSO ( $n=3$ mice, $n=46$ cardiomyocytes examined over 3 independent experiments), ark $^{+/+}$myocardial infarction PTL ( $n=3$ mice, $n=67$ cardiomyocytes examined over 3 independent experiments), Mark4 $^{-/-}$ myocardial infarction DMSO $(n=3$ mice, $n=55$ cardiomyocytes examined over 3 independent experiments) and $\mathrm{Mark}^{-/-}$myocardial infarction PTL $(n=3$ mice, $n=64$ cardiomyocytes examined over 3 independent experiments). $\mathbf{j}$, Colour denotation of samples. $\mathbf{k}$, Resting sarcomere length. I, Sarcomere peak shortening. $\mathbf{m}-\mathbf{o}$, Average sarcomere shortening traces. p. Pooled data of contraction velocity and relaxation velocity. q-s, Average velocity traces (dSL/dT). c, k, l, p, The box bounds represent the 25 th and 75 th percentiles, the middle line shows the median, the whiskers show the minimum and maximum and individual cardiomyocytes are shown as circles. $\mathbf{c}, \mathbf{k}, \mathbf{l}, \mathbf{p}$, Two-way ANOVA test with Bonferroni post hoc correction for multiple comparisons; $P$ values are indicated. 


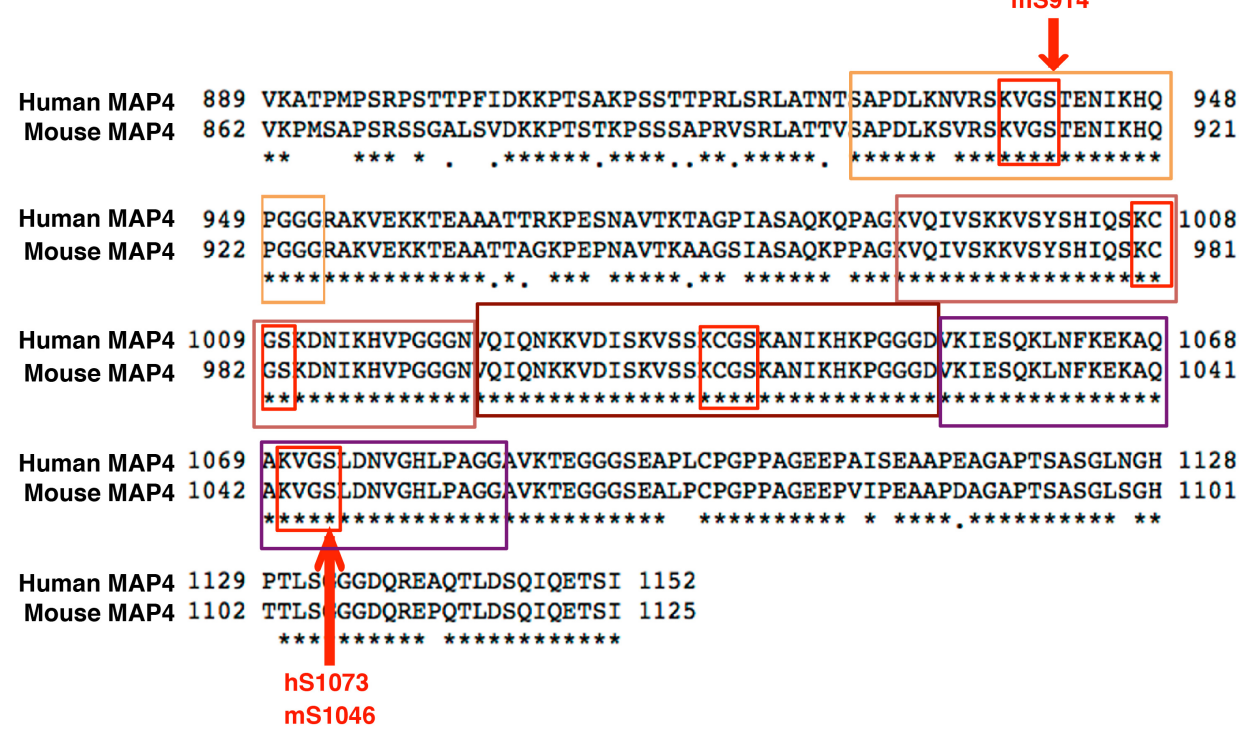

b
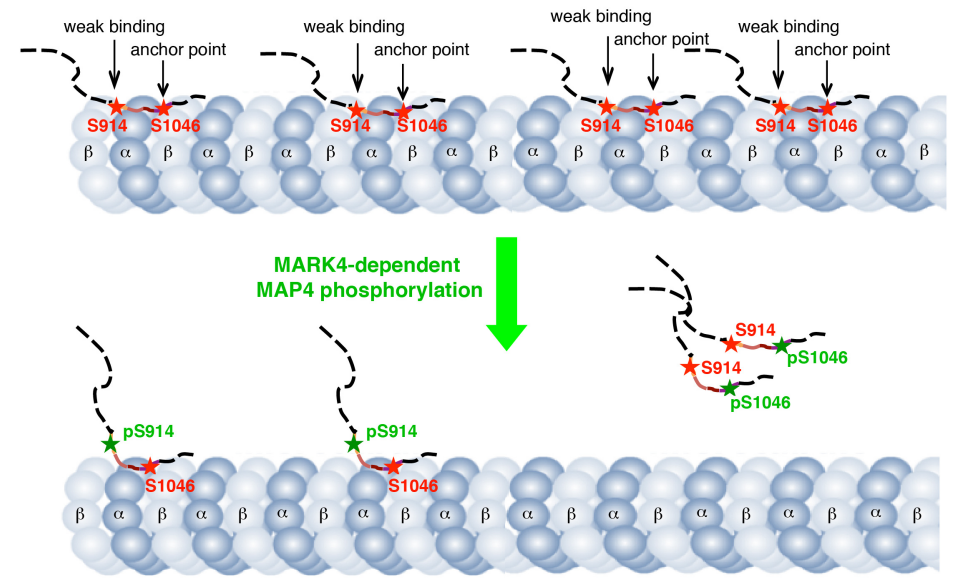

C

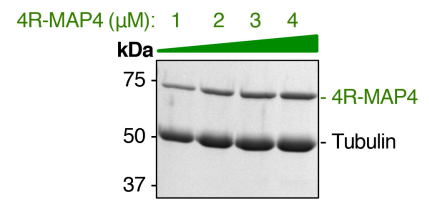

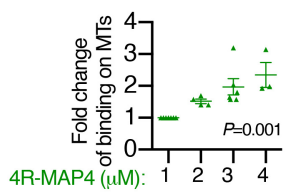

f

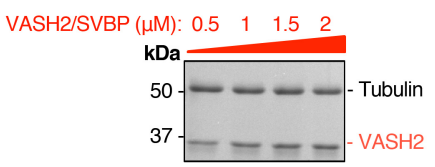

Extended Data Fig. 6 | The association of MAP4 or VASH2 with polymerized microtubules. a, Protein sequence alignment between human MAP4 (NP002366) and mouse MAP4 (NP001192259). KXGS motifs (highlighted with red frames) within the tubulin-binding repeats (highlighted with yellow, brown, dark brown and purple frames) of MAP4 are MARK4 substrate sites. S941 of human MAP4 (S914 of mouse MAP4) and S1073 of human MAP4 (S1046 of mouse MAP4) are conserved phosphorylation sites within the KXGS motifs. b, Schematic illustration of the possible association between MAP4 and microtubules before or after MARK4-dependent phosphorylation. Unphosphorylated MAP4 binds to microtubules. After MARK4-dependent phosphorylation of S914 of mouse MAP4 at the microtubule weak binding site, MAP4 makes allosteric changes. After MARK4-dependent phosphorylation of S1046 of mouse MAP4 at the microtubule anchor site, MAP4 detaches from

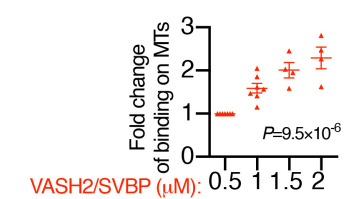

microtubules. c, Representative gel image of 4R-MAP4 (1-4 $\mu \mathrm{M})$ binding to the polymerized microtubules $(5 \mu \mathrm{M})$ in a microtubule co-sedimentation assay. d, Quantification of the binding shown in c. $n=7$ samples examined over 3 independent experiments $(1 \mu \mathrm{M}) ; n=4$ samples examined over 3 independent experiments $(2 \mu \mathrm{M}) ; n=6$ samples examined over 3 independent experiments $(3 \mu \mathrm{M}) ; n=3$ samples examined over 3 independent experiments $(4 \mu \mathrm{M})$. e, Representative gel image of VASH2-SVBP $(0.5-2 \mu \mathrm{M})$ binding to the polymerized microtubules $(2.5 \mu \mathrm{M})$ in a microtubule co-sedimentation assay. |f, Quantification of the binding shown in e. $n=7$ samples examined over 5 independent experiments $(0.5 \mu \mathrm{M}) ; n=7$ samples examined over 5 independent experiments $(1 \mu \mathrm{M}) ; n=4$ samples examined over 3 independent experiments $(1.5 \mu \mathrm{M}) ; n=4$ samples examined over 3 independent experiments $(2 \mu \mathrm{M})$. d,f, Data are mean \pm s.e.m.; one-way ANOVA; $P$ values are indicated. 


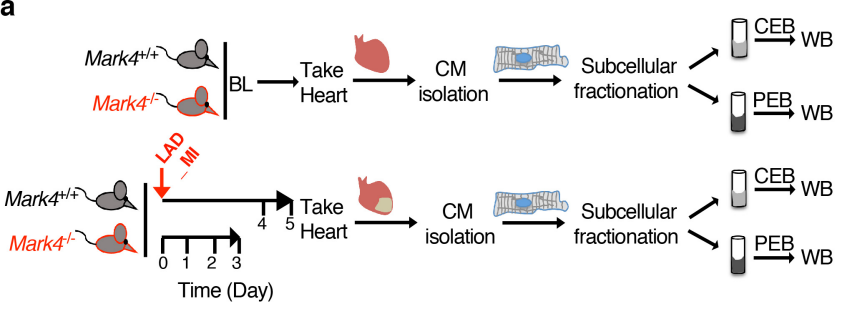

C

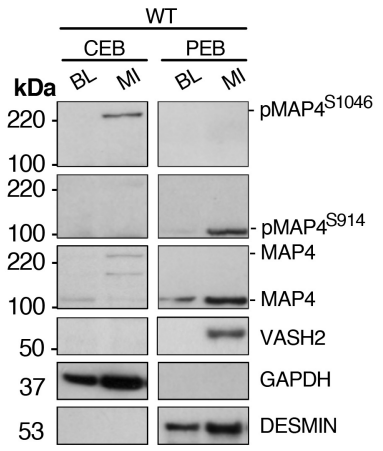

d

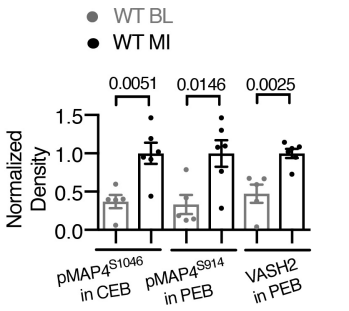

b

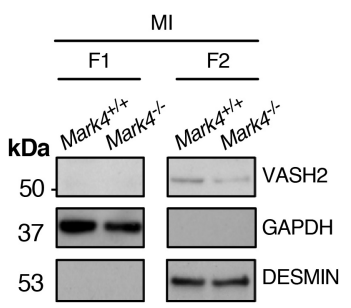

e

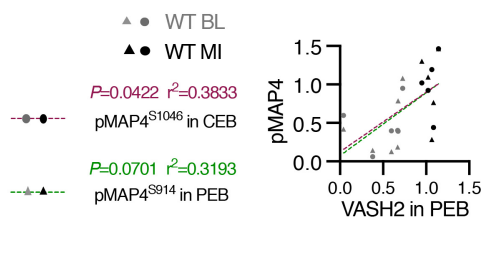

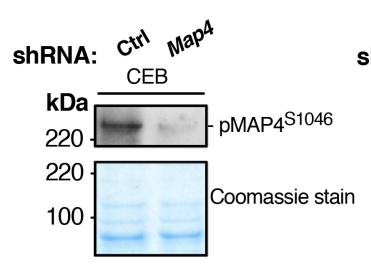

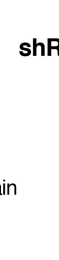

h
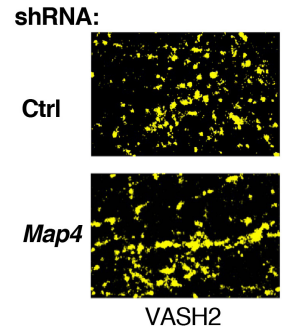
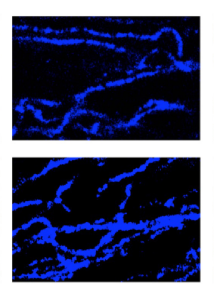

a-tub

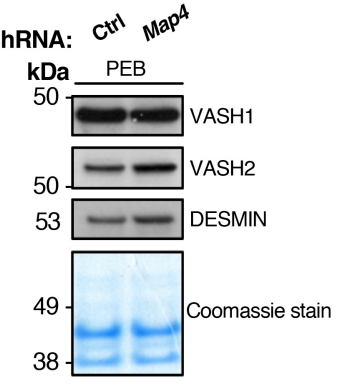

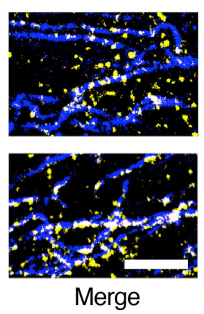

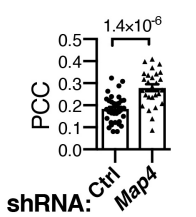

shRNA:

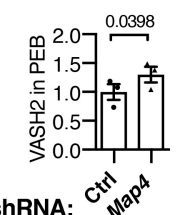

Extended Data Fig. 7 |Association of VASH2 with microtubules in cardiomyocytes before and myocardial infarction, and the effect of MAP4 knockdown.a, Subcellular fractionation of primary cardiomyocytes isolated from mice at baseline or after myocardial infarction. Western blotting of the fractions from CEB or PEB. b, Representative western blots of the free-tubulin fraction (F1) and extraction from the stable pellet fraction (F2) obtained using a conventional fractionation method. $\mathbf{c}-\mathbf{e}$, Western blots of CEB or PEB fractions of wild-type (WT) cardiomyocytes at baseline or after myocardial infarction. c, Representative western blots (derived from the same experiment). d, Quantification of pMAP4(S1046) in CEB, pMAP4(S914) in PEB and VASH2 levels in PEB ( $n=5$ mice at baseline, $n=6$ mice after myocardial infarction, blots were processed in parallel). e, Correlation between VASH2 level in the PEB fraction and pMAP4 levels. $\mathbf{f}-\mathbf{i}$, Wild-type cardiomyocytes after myocardial infarction transduced with adenovirus-mediated Map4 or control shRNA. f, Representative western blots of CEB or PEB fraction, and Coomassie-stained gels loaded with the same amounts of proteins. g, Quantification of VASH2 levels in PEB ( $n=3$ mice examined over 3 experiments per group). h, i, STED images of VASH 2 and $\alpha$-tubulin in the cardiomyocytes after knocking down MAP4.h, Representative images. Scale bar, $2 \mu \mathrm{m} . \mathbf{i}$, Pearson correlation coefficient of VASH 2 and $\alpha$-tubulin signals, percentage of VASH 2 signals on the polymerized microtubules and percentage of VASH2 signals off the polymerized microtubules, in the following groups: control shRNA ( $n=2$ mice, $n=35$ cardiomyocytes examined over 2 independent experiments) and Map4 shRNA ( $n=2$ mice, $n=27$ cardiomyocytes examined over 2 independent experiments).d, $\mathbf{g}, \mathbf{i}$, Data are mean \pm s.e.m.; two-tailed unpaired $t$-test. e, Two-tailed correlation test. $P$ values are indicated. 


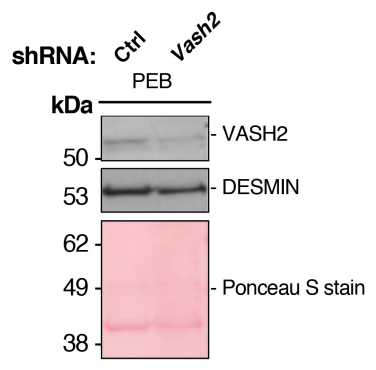

b

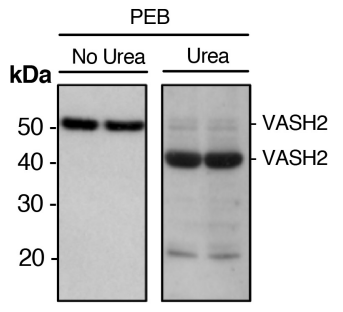

C

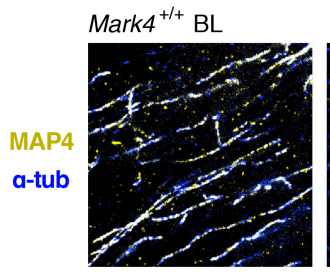

e

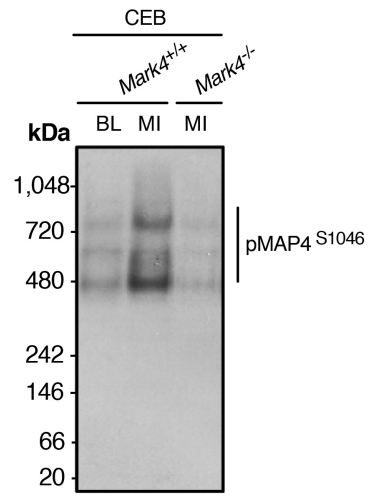

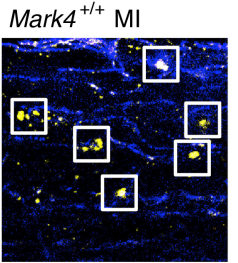

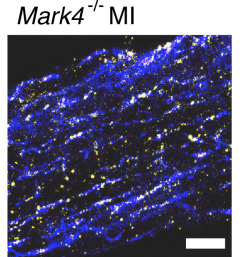

d

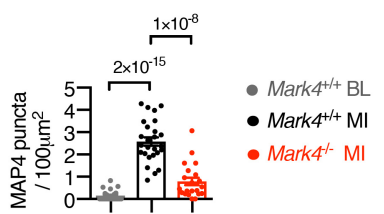

g
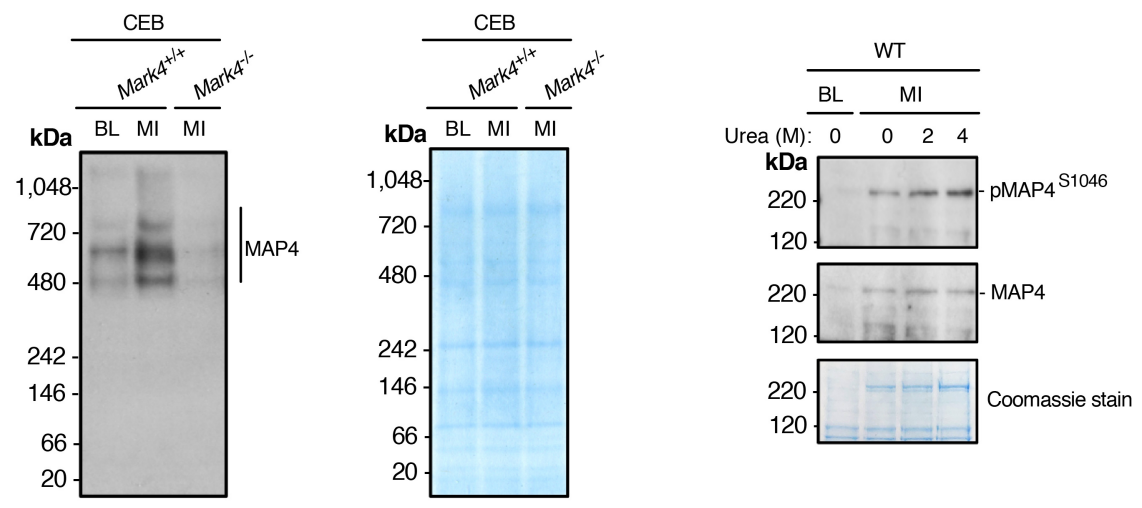

h

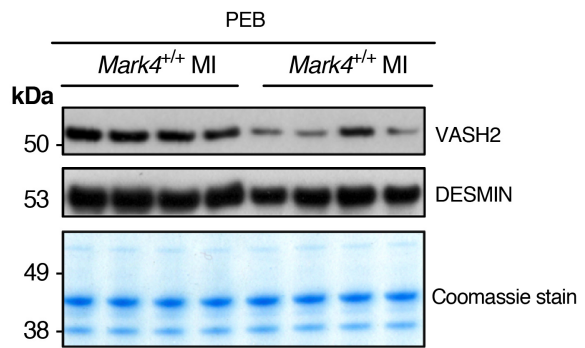

Extended Data Fig. 8 | The status of VASH2 and MAP4 in cardiomyocytes before and after myocardial infarction. a, Subcellular fractionation of wild-type cardiomyocytes, isolated from mice after myocardial infarction and transduced with adenovirus-mediated Vash2 or control shRNA. Representative western blots of fraction in PEB, with the same membrane stained with Ponceau S. b, Representative western blot of PEB extractions denatured in the presence or absence of urea from cardiomyocytes after myocardial infarction. c, d, STED images of MAP4 and $\alpha$-tubulin in cardiomyocytes of $\mathrm{Mark}^{-/-}$or control mice at baseline or after myocardial infarction. c, Representative images. Oligomerized puncta are indicated within the square frames. Scale bar, $2 \mu \mathrm{m}$. d, Quantification of the presence of the MAP4 oligomerized puncta in the following groups: Mark $^{+/+}$baseline ( $n=2$ mice, $n=22$ cardiomyocytes examined over 2 independent experiments), Mark $^{+/+}$myocardial infarction ( $n=2$ mice, $n=26$ cardiomyocytes examined over 2 independent experiments) i

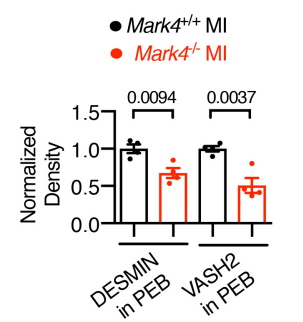

j

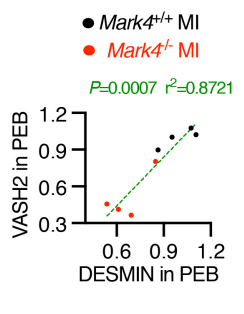

and $\mathrm{Mark4}^{-/-}$myocardial infarction ( $n=2$ mice, $n=21$ cardiomyocytes examined over 2 independent experiments).e, f, Western blot of native gels loaded with samples in CEB of cardiomyocytes isolated at baseline or after myocardial infarction.e, The presence of pMAP4(S1046) and total MAP4 is indicated.f, Coomassie-stained native gel loaded with the same amounts of proteins as used in e.g, Western blot of the CEB fraction denatured in the presence of urea, with a Coomassie-stained denaturing gel loaded with the same amounts of protein. h, Western blot of fractions in PEB of cardiomyocytes isolated from $\mathrm{Mark4}^{-/-}$or control mice after myocardial infarction, with a Coomassie-stained gel loaded with the same amounts of proteins. i, Quantification of VASH2 and desmin levels in PEB fraction ( $n=4$ mice per group).j, Correlation between desmin and VASH2 levels in PEB.d, i, Data are mean \pm s.e.m.; two-tailed unpaired $t$-test.j, Two-tailed correlation test. $P$ values are indicated. 


\section{Article}

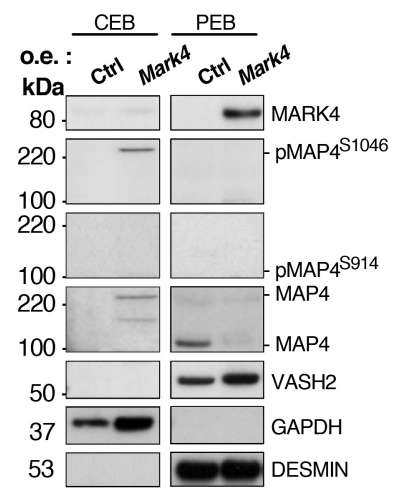

d

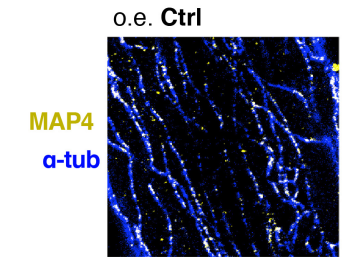

o.e. Mark4

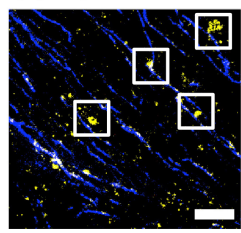

Extended Data Fig. 9 | MARK4 overexpression regulates MAP4 phosphorylation, and the presence of MAP4 oligomers in the cytosolic fraction. a-c, Subcellular fractionation of wild-type cardiomyocytes transduced with adenovirus to overexpress Mark4 or a null control. a, Representative western blots of fractions in CEB or PEB (derived from the same experiment). b, Quantification of pMAP4(S1046) in CEB and VASH2 level in PEB ( $n=5$ mice per group, blots were processed in parallel).c Correlation between VASH2 level in the PEB fraction and pMAP4 levels.d, e, STED images of
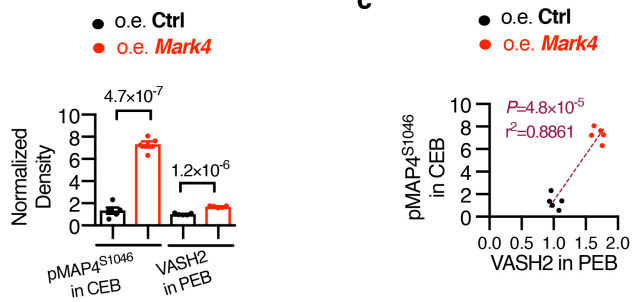

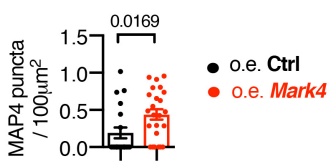

MAP4 and $\alpha$-tubulin in wild-type cardiomyocytes at baseline transduced with adenovirus to overexpress Mark4 or a null control. d, Representative images. Scale bar, $2 \mu \mathrm{m}$. e, Quantification of MAP4 oligomerized puncta in the following groups: overexpression of control ( $n=2$ mice, $n=20$ cardiomyocytes examined over 2 independent experiments) and overexpression of $\operatorname{Mark} 4$ ( $n=2$ mice, $n=24$ cardiomyocytes examined over 2 independent experiments). d, e, Data are mean \pm s.e.m.; two-tailed unpaired $t$-test.c, Two-tailed correlation test. $P$ values are indicated. 
a

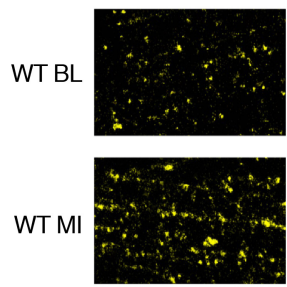

VASH2
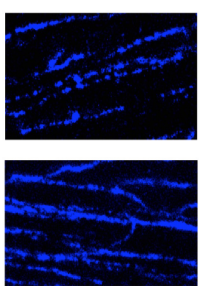

a-tub
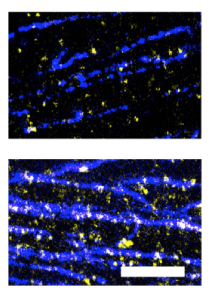

Merge b

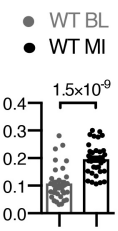

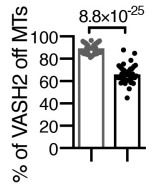

c

d

- Mark4 ${ }^{+/+} \mathrm{MI}$

- Mark4 $4^{-} \mathrm{MI}$

Mark4t- MI

|י⿴囗十)

e

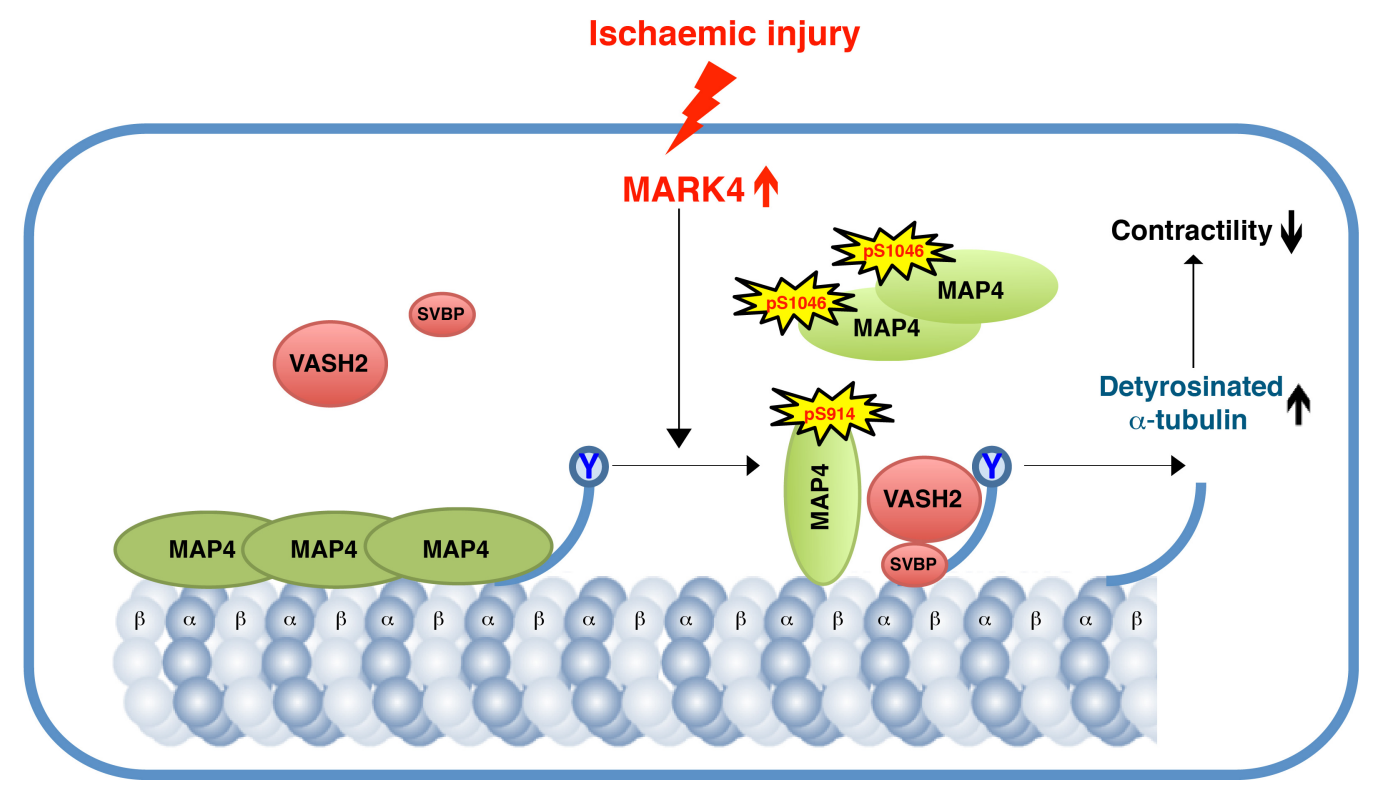

Extended Data Fig. 10 | VASH2 status in cardiomyocytes before and after myocardial infarction, and the schematic summary of the results. a,b, STED images of VASH 2 and $\alpha$-tubulin in wild-type cardiomyocytes at baseline or after myocardial infarction. a, Representative images. Scale bar, $2 \mu \mathrm{m}$. b. Pearson correlation coefficient of VASH 2 and $\alpha$-tubulin signals, percentage of VASH2 signals on the polymerized microtubules and percentage of VASH2 signals off the microtubules in the following groups: wild-type baseline ( $n=4$ mice, $n=38$ cardiomyocytes examined over 2 independent experiments) and wild-type myocardial infarction ( $n=38$ cardiomyocytes of $n=6$ mice, $n=38$ cardiomyocytes examined over 3 independent experiments). c, Real-time PCR of cardiomyocytes after myocardial infarction from the following groups: $\mathrm{Mark4}^{+/+}$myocardial infarction $(n=5 \mathrm{mice})$ and $\mathrm{Mark}^{-/-}$ myocardial infarction ( $n=6$ mice). d, Quantification of VASH2 mean fluorescence intensity (MFI) within the cell area (region of interest (ROI)) using the STED images from the following groups: $\mathrm{Mark}^{+/+}$myocardial infarction ( $n=6$ mice, $n=38$ cardiomyocytes examined over 3 independent experiments) and $\mathrm{Mark}^{-/-}$myocardial infarction ( $n=6$ mice, $n=47$ cardiomyocytes examined over 3 independent experiments). b-d, Data are mean \pm s.e.m.; two-tailed unpaired $t$-test. $P$ values are indicated. e, A working model for the MARK4-dependent regulation of microtubule detyrosination after myocardial infarction. After ischaemic injury, increased MARK4 phosphorylates MAP4 at its KXGS motifs. Phosphorylated MAP4 either changes its conformation on the polymerized microtubules or detaches itself from the polymerized microtubules to form oligomerized MAP4 structures in the cytosol. The phosphorylation of MAP4 by MARK4 allows for space access of VASH 2 to the polymerized microtubules, thereby promoting $\alpha$-tubulin detyrosination. As a consequence, the increased level of detyrosinated microtubules causes a reduction in the contractile function of the cardiomyocyte. 


\section{Reporting Summary}

Nature Research wishes to improve the reproducibility of the work that we publish. This form provides structure for consistency and transparency

in reporting. For further information on Nature Research policies, see our Editorial Policies and the Editorial Policy Checklist.

\section{Statistics}

For all statistical analyses, confirm that the following items are present in the figure legend, table legend, main text, or Methods section.

n/a Confirmed

$\bigotimes$ The exact sample size $(n)$ for each experimental group/condition, given as a discrete number and unit of measurement

$\bigotimes$ A statement on whether measurements were taken from distinct samples or whether the same sample was measured repeatedly

The statistical test(s) used AND whether they are one- or two-sided

Only common tests should be described solely by name; describe more complex techniques in the Methods section.

$\bigotimes \square$ A description of all covariates tested

$\square$ \A description of any assumptions or corrections, such as tests of normality and adjustment for multiple comparisons

$\square$ A full description of the statistical parameters including central tendency (e.g. means) or other basic estimates (e.g. regression coefficient)

$\triangle$ AND variation (e.g. standard deviation) or associated estimates of uncertainty (e.g. confidence intervals)

$\varnothing$ For null hypothesis testing, the test statistic (e.g. $F, t, r$ ) with confidence intervals, effect sizes, degrees of freedom and $P$ value noted Give $P$ values as exact values whenever suitable.

$\bigotimes \square$ For Bayesian analysis, information on the choice of priors and Markov chain Monte Carlo settings

$\bigotimes \square$ For hierarchical and complex designs, identification of the appropriate level for tests and full reporting of outcomes

$\bigotimes$ Estimates of effect sizes (e.g. Cohen's $d$, Pearson's $r$ ), indicating how they were calculated

Our web collection on statistics for biologists contains articles on many of the points above.

\section{Software and code}

Policy information about availability of computer code

Data collection VisualSonics Vevo 3100 was used for echocardiography data collection; lonWizard 7.4 was used for Cardiomyocyte contractility and calcium data collection; BD FACSDiva Software 6.0 was used for the flow cytometry data collection; LightCycler 480 software release 1.5.0.39 was used for real time PCR data collection; Leica Application Suite Advanced Fluorescence (LAS AF) 2.4.0 build 6254 was used for IHC and histology data collection; Leica Application Suite Advanced Fluorescence (LAS AF) 2.7.3.9723 was used for the confocal microscopy data collection; STED image data were collected with a custom program written in National Instrument (NI) LabVIEW 2014 64-bit, NI FPGA Module and NI Vision Development Module. Custom STED microscope control software used for data collection in this study is available on GitHub and can be accessed online at https://github.com/Gurdon-Super-Res-Lab/STED-Control

Data analysis GraphPad Prism 7.05 and Microsoft Excel (version 2102) was used for statistics data analysis, and GraphPad 9.1.0 (216) was used for violin plots; Vevo LAB3.1.1. was used for echocardiography data analysis; IonWizard 7.4 was used for cardiomyocyte contractility and calcium data analysis; FlowJo (v10) was used for flow cytometry data analysis; Image J (v2.0) was used for western blot band density analysis, histology \& IHC image analysis, confocal image analysis, and some of STED image analysis; A customized algorithm, available in GitHub (https:// github.com/zhaoaite/dynamic_thresholding_algorithm), was used for STED image analysis. 
Policy information about availability of data

All manuscripts must include a data availability statement. This statement should provide the following information, where applicable:

- Accession codes, unique identifiers, or web links for publicly available datasets

- A list of figures that have associated raw data

- A description of any restrictions on data availability

All the associated raw data presented in this paper are available from the corresponding author upon reasonable request. Source data are provided with this paper.

\section{Field-specific reporting}

Please select the one below that is the best fit for your research. If you are not sure, read the appropriate sections before making your selection. \Life sciences Behavioural \& social sciences Ecological, evolutionary \& environmental sciences

For a reference copy of the document with all sections, see nature.com/documents/nr-reporting-summary-flat.pdf

\section{Life sciences study design}

All studies must disclose on these points even when the disclosure is negative.

Sample size Sample sizes for in vivo experiments were based on knowledge of the intra-group variation and pilot conducted experiments in which statistically significant differences were observed between groups. Samples size was also determined as reasonable number of animals allowing statistical analysis while complying with the 3Rs rule on reducing, replacing and refining the use of animals for scientific purpose. The sample size ( $n$ ) of each experiment is provided in the figure legends.

Data exclusions Data were excluded if the LAD surgeries failed (mice were not recovered within 24h post-MI or underwent unsuccessful LAD ligation). Outlier exclusion was applied to Masson staining and TTC staining data, and samples with small infarct/scar size less than $35 \%$ (due to failed LAD ligation or anatomy variant) were excluded from data. STED images with no good focus on the linear microtubule structures were excluded from the STED image analysis.

Replication In vivo findings at early time points post-MI were replicated at least 3 times (batches) in independent experiments. All the ex vivo and in vitro experiments were biologically \& independently replicated as noted in text, figure legends and methods.

Randomization All studies were performed with randomization when necessary. Mice were age, gender, genetic background-matched littermates between groups. Mice were randomly assigned prior to surgery, bone marrow transplantation, and other interventions. Cardiomyocytes were randomly selected within each genotype or treatment group in contractility experiments and imaging experiments. Cardiomyocytes were randomly assigned with different treatments or transductions. Randomization does not apply to co-sedimentation assay.

Blinding Investigators were blinded to the genotypes when performing surgeries, echocardiography measurements, confocal imaging, and STED imaging. All data analysis on above experiments, and histological analysis were conducted in a blinded manner. Measurement of cTnl and inflammatory cytokines were conducted by core facility researchers without knowing the genotypes. Microtubule co-sedimentation assay, real time PCR, and the ex vivo experiments (contractility and calcium measurements, fractionation assays, and western blotting ) were not blinded due to logistical issues, but all the procedures and analysis were equal in different groups in those experiments.

\section{Reporting for specific materials, systems and methods}

We require information from authors about some types of materials, experimental systems and methods used in many studies. Here, indicate whether each material, system or method listed is relevant to your study. If you are not sure if a list item applies to your research, read the appropriate section before selecting a response.

Materials \& experimental systems

\begin{tabular}{l|l}
\multicolumn{2}{l}{ Methods } \\
\hline n/a & Involved in the study \\
\hline & $\square$ ChIP-seq \\
$\square$ & $\bigotimes$ Flow cytometry \\
$\square$ & $\square$ MRI-based neuroimaging
\end{tabular}

$\mathrm{n} / \mathrm{a}$ Involved in the study

$\square$ \ Antibodies

Х $\square$ Eukaryotic cell lines

\ $\square$ Palaeontology and archaeology

\ $\square$ MRI-based neuroimaging

$\square$ \ Animals and other organisms

$\bigotimes \square$ Human research participants

Х $\square$ Clinical data

$\bigotimes \square$ Dual use research of concern 
MARK4 Ab, CST, 4834S, 3, 1:1000 WB:

MARK4 Ab, Abcam, ab12426, GR3197381-5, 1:200 IF, 1:200 IHC;

Detyrosinated $\alpha$-tubulin Ab, Abcam, ab48389, GR3309909-1, 1:1000 WB, 1:200 IF

$\alpha$-tubulin Ab (DM1A), CST, 3873S, 12, 1:1000 WB, 1:200 IF, 1:200 STED;

MAP4 (phospho S1073) Ab, Abnova, PAB15916, 1021, 1:1000 WB;

MAP4 (phospho 5941) Ab, Abcam, ab56087, GR324863-2, 1:1000 WB

MAP4 Ab, Abcam, ab245578, GR3259936-1, 1:1000 WB, 1:200 STED;

VASH1 Ab, Abcam, ab199732, GR3224901-2, 1:1000 WB;

VASH2 Ab, Abcam, ab224723, GR3199655-27, 1:1000 WB, 1:200 STED;

GAPDH Ab(D16H11), CST, 5174S, 6, 1:1000 WB;

DESMIN Ab, R\&D, AF3844, YIL0617121, 1:1000 WB;

TTL Ab, Proteintech, 13618-1-AP, 1:1000 WB;

Polyglutamylated $\alpha$-Tubulin Ab (GT335), AdipoGen, AG-20B-0020-C100, A27791601, 1:1000 WB;

Acetylated $\alpha$-Tubulin Ab, Santa Cruz Biotechnology, sc23950, C1813, 1:1000 WB;

Rabbit IgG isotype control, Novus Biologicals, NB810-56910, P120-101-11, 1:1000 IHC, 1:1000 IF;

APC anti-mouse CD45 Ab (30-F11), BioLegend, 103112, B210891, 1:200 IF, 1:100 FC;

Brilliant Violet 605 anti-mouse CD3 Ab (17A2), BioLegend, 100237, 1:100 FC

FITC anti-mouse CD19 Ab (1D3), Biosciences, 553785, 1:100 FC;

PECY7 anti-mouse CD11c Ab (N418), Biolegend, 117318, 1:100 FC

AF488 anti-mouse CD11b Ab (M1/70), Biolegend, 101217, 1:100 FC;

Pacific blue anti-mouse Ly6G Ab (1A8), Biolegend, 127612, 1:100 FC;

PE anti-mouse F4/80 Ab (BM8), Biolegend, 123110, 1:100 FC;

Biotinylated anti-rabbit secondary antibody, Abcam, ab6720, 1:800 IHC.

Atto 594 goat anti-Rabbit IgG, Sigma, 77671, 1:500 STED;

Atto 647N goat anti-mouse IgG, Sigma 50185, 1:500 STED;

AF488 donkey anti-rabbit IgG, Invitrogen, A21206, 1981155, 1:200 IF;

AF647 goat anti-mouse IgG, Invitrogen, A21236, 1654338, 1:200 IF;

AF647 goat anti-rat IgG; Invitrogen, A21247, 2999156, 1:200 IF;

Goat Anti-Mouse IgG H\&L (HRP), Abcam, ab205719, GR3279214-3, 1:100,000 WB

Goat Anti-Rabbit IgG H\&L (HRP), Abcam, ab205718, GR3307521-1, 1:100,000 WB;

Polyclonal rabbit anti goat IgG (HRP), Dako, P0160, 00072616, 1:10,000 WB.

Antibodies were validated based on information provided by the vendors, as listed below.

MARK4 Ab(CST, 4834S, https://www.cellsignal.com/products/primary-antibodies/mark4-antibody/4834) used in WB was validated by the absent detection of specific signals using Mark4-/-samples, and the presence of specific detection with heterologous overexpression of MARK4.

MARK4 Ab (Abcam, ab12426, https://www.abcam.com/mark4-antibody-ab124267.html) used in IF \& IHC was validated by the absent detection of specific signals using Mark4-/-samples, and by using appropriate IgG isotype control.

VASH2 Ab (Abcam, ab224723, https://www.abcam.com/vash2-antibody-ab224723.html) used in WB was validated by using samples with specific knocking-down of VASH2. The antibody used in STED was validated by using appropriate IgG isotype control.

Detyrosinated $\alpha$-tubulin Ab, https://www.abcam.com/detyrosinated-alpha-tubulin-antibody-microtubule-marker-ab48389.htm

$\alpha$-tubulin Ab, https://www.cellsignal.com/products/primary-antibodies/a-tubulin-dm1a-mouse-mab/3873

MAP4 (phospho \$1073) Ab, http://www.abnova.com/products/products_detail.asp?catalog_id=PAB15916

MAP4 (phospho S941) Ab, https://www.abcam.com/map4-phospho-s941-antibody-carboxyterminal-end-ab56087.html

MAP4 Ab, https://www.abcam.com/map4-antibody-ab245578.htm

VASH1 Ab, https://www.abcam.com/vash1-antibody-epr17420-ab199732.html

VASH2 Ab, https://www.abcam.com/vash2-antibody-ab224723.hatml

GAPDH Ab(D16H11), https://www.cellsignal.com/products/primary-antibodies/gapdh-d16h11-xp-rabbit-mab/5174

DESMIN Ab, https://www.novusbio.com/products/desmin-antibody_af3844

TTL Ab, http://www.ptgcn.com/products/TTL-Antibody-13618-1-AP.htm 
Polyglutamylated $\alpha$-Tubulin Ab (GT335), https://adipogen.com/storeconfig/choose/store?destination=ag-20b-0020-antipolyglutamylation-modification-mab-gt335.html

Acetylated $\alpha$-Tubulin Ab, https://www.scbt.com/p/acetylated-alpha-tubulin-antibody-6-11b-1? gclid=CjwKCAjwpKCDBhBPEiwAFgBzjzvZolyuC_qgZ7seUiq7PzUOZjM912087g00-yGuHOlw33wovOON2BoCE5AQAvD_BwE

Rabbit IgG isotype control, https://www.novusbio.com/products/igg-isotype-control_nb810-56910

APC anti-mouse CD45 Ab(30-F11), https://www.biolegend.com/en-us/products/apc-anti-mouse-cd45-antibody-97

Brilliant Violet 605 anti-mouse CD3 Ab (17A2), https://www.biolegend.com/fr-fr/products/brilliant-violet-605-anti-mouse-cd3antibody-8503

FITC anti-mouse CD19 Ab (1D3), https://www.bdbiosciences.com/eu/applications/research/stem-cell-research/hematopoietic-stemcell-markers/mouse/negative-markers/fitc-rat-anti-mouse-cd19-1d3/p/553785

PECY7 anti-mouse CD11c Ab (N418), https://www.biolegend.com/ja-jp/products/pe-cyanine7-anti-mouse-cd11c-antibody-3086

AF488 anti-mouse CD11b Ab (M1/70), https://www.biolegend.com/en-gb/products/alexa-fluor-488-anti-mouse-human-cd11bantibody-2700

Pacific blue anti-mouse Ly6G Ab (1A8), https://www.biolegend.com/en-gb/products/pacific-blue-anti-mouse-ly-6g-antibody-6082

PE anti-mouse F4/80 Ab (BM8), https://www.biolegend.com/en-gb/products/pe-anti-mouse-f4-80-antibody-4068

\section{Animals and other organisms}

Policy information about studies involving animals; ARRIVE guidelines recommended for reporting animal research

Laboratory animals

All mice were on a C57BL/6 background and housed under standard conditions of temperature $\left(18-23^{\circ} \mathrm{C}\right)$ and humidity $(40-60 \%)$ with a 12-hour light/dark cycle. Mark4-/- mice were kindly provided by Prof Yuguan Shi(Barshop Institute), and Mutant Mouse Resource and Research Center (MMRRC, University of California, Davis); $\alpha \mathrm{MHC}-\mathrm{mcm}+$ - Cre mice were originally from the Jackson Laboratory;

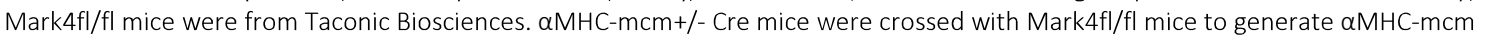
$+/$ - Cre; Mark4fl/fl. All mice were used at eight to ten-week old. Mice used in Fig 1d, 1e, 2a, 2b, 2c ,2d, 2e, and Supplementary table $1 \&$ table 2 are females, both male and female mice were used in the other experiments.

Wild animals

No wild-animals were used in the study.

Field-collected samples

No field-collected samples were used in the study.

Ethics oversight

All experiments were approved by the Home Office, UK, and were performed under PPL PA4BDF775.

Note that full information on the approval of the study protocol must also be provided in the manuscript.

\section{Flow Cytometry}

\section{Plots}

Confirm that:

ХThe axis labels state the marker and fluorochrome used (e.g. CD4-FITC).

\The axis scales are clearly visible. Include numbers along axes only for bottom left plot of group (a 'group' is an analysis of identical markers).

\All plots are contour plots with outliers or pseudocolor plots.

\A numerical value for number of cells or percentage (with statistics) is provided.

\section{Methodology}

Sample preparation

The method of sample preparation is available in the Methods.

Instrument

FACS LSR II Fortessa (BD Biosciences).

Software

Flow cytometry data were collected using BD FACSDiva Software 6.0, and flow cytometry data were analyzed using FlowJo software (v10).

Cell population abundance

Cardiac CD45+ immune cells were around $0.3-0.8 \%$ of the single live cells in sham group, and around $1.3-6 \%$ of the single live cells in the MI group; Cardiac CD45+ CD11c high cells were around 2-4\% of the CD45+ cells in the sham group, and around $7-16 \%$ of the CD45+ cells in the MI group; Cardiac T cells were around $7-20 \%$ of the CD $45+$ cells in the sham group, and 
around $8-38 \%$ pf the CD45+ cells in the MI group; Cardiac B cells were around $30-40 \%$ of the CD45+cells in the sham group, and around $30-60 \%$ of the CD $45+$ cells in the MI group; Cardiac neutrophils were around $6-8 \%$ of the CD $45+$ cells in the sham group, and around $11-40 \%$ of the CD45+ cells in the MI group; Cardiac macrophages were around $8-13 \%$ of the CD45+ cells in the sham group, and around $26-50 \%$ of the CD45+ cells in the MI group.

Gating strategy

The relevant gating strategies are shown in Supplementary Figure 2.

\ Tick this box to confirm that a figure exemplifying the gating strategy is provided in the Supplementary Information. 